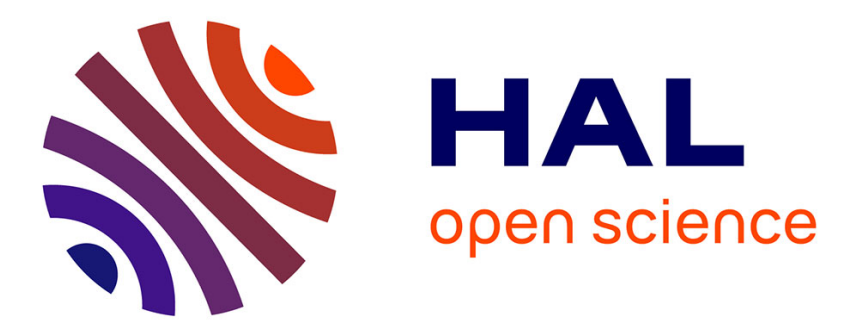

\title{
Monitoring conformational changes during the catalytic cycle of OpuAA, the ATPase subunit of the ABC-transporter OpuA from Bacillus subtilis
}

Carsten Horn, Stefan Jenewein, Britta Tschapek, Werner Bouschen, Sabine Metzger, Erhard Bremer, Lutz Schmitt

\section{To cite this version:}

Carsten Horn, Stefan Jenewein, Britta Tschapek, Werner Bouschen, Sabine Metzger, et al.. Monitoring conformational changes during the catalytic cycle of OpuAA, the ATPase subunit of the ABC-transporter OpuA from Bacillus subtilis. Biochemical Journal, 2008, 412 (2), pp.233-244. 10.1042/BJ20071443 . hal-00478899

\section{HAL Id: hal-00478899 https://hal.science/hal-00478899}

Submitted on 30 Apr 2010

HAL is a multi-disciplinary open access archive for the deposit and dissemination of scientific research documents, whether they are published or not. The documents may come from teaching and research institutions in France or abroad, or from public or private research centers.
L'archive ouverte pluridisciplinaire HAL, est destinée au dépôt et à la diffusion de documents scientifiques de niveau recherche, publiés ou non, émanant des établissements d'enseignement et de recherche français ou étrangers, des laboratoires publics ou privés. 


\section{Monitoring conformational changes during the catalytic cycle of OpuAA, the ATPase subunit of the ABC-transporter OpuA from Bacillus subtilis}

Carsten Horn ${ }^{1, *,+}$, Stefan Jenewein ${ }^{1, *}$, Britta Tschapek ${ }^{1}$, Werner Bouschen ${ }^{2}$, Sabine Metzger $^{2}$, Erhard Bremer ${ }^{3} \&$ Lutz Schmitt ${ }^{1, 末}$

1: Institute of Biochemistry, Heinrich Heine University Duesseldorf, Universitaetsstr. 1, 40225 Duesseldorf, Germany.

2: Biological and Medical Research Center, Heinrich Heine University, Duesseldorf, Universitaetsstr. 1, 40225 Duesseldorf, Germany.

3. Laboratory for Microbiology, Department of Biology, Philipps University Marburg, Karlvon-Frisch Str., 35032 Marburg, Germany.

+: Present address: CellGenix Technology Transfer GmbH, Am Flughafen 16, 79108 Freiburg, Germany.

*: $\quad$ Both authors contributed equally to this work

†. Corresponding author:

Lutz Schmitt

Institute of Biochemistry, Heinrich Heine University Duesseldorf, Universitaetsstr. 1, 40225 Duesseldorf, Germany.

Phone: $\quad+49(0) 211-81-10773$

Fax: $\quad+49(0) 211-81-15310$

E-mail. $\quad$ lutz.schmitt@uni-duesseldorf.de 


\begin{abstract}
The ABC-transporter OpuA is one of five membrane transport systems in B. subtilis that mediate osmoprotection by importing compatible solutes. Just like all bacterial and archaeal ABC-transporters that catalyze the import of substrates, OpuA is composed of an ATPase subunit (OpuAA), a transmembrane subunit (OpuAB) and an extracellular substrate binding protein (OpuAC). In contrast to many well-known ABC-ATPases, OpuAA is not only composed of a catalytic and helical domain but also of an accessory domain located at its Cterminus. The paradigm of such an architecture is MalK, the ABC-ATPase of the maltose importer of $E$. coli, for which detailed structural and functional information is available. Here we have applied solution FRET techniques using two single cysteine mutants to obtain initial structural information on the architecture of the OpuAA dimer in solution. Analyzing our results in detail and comparing it with the existing MalK structures revealed that the catalytic and helical domain adopted an arrangement similar to MalK, while profound differences in the three-dimensional orientation of the accessory domain, which contains two CBS domains, were observed. These results shed new light on the role of this accessory domain present in a certain subset of ABC-ATPase in the fine-tuning of three-dimensional structure and biological function.
\end{abstract}

Running title: FRET analysis of OpuAA

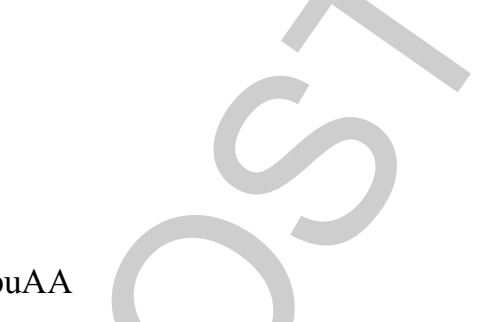

Key words: ABC-ATPase, osmoprotection, conformational changes, dimer, FRET

\begin{abstract}
Abbreviations: ABC: ATP-binding cassette; B. subtilis: Bacillus subtilis; BSA: buried surface area; CBS: cystathionine $\beta$-synthase; FRET: Förster resonance energy transfer; $L$. lactis: Lactococcus lactis; NBD: nucleotide-binding domain or subunit; OG: Oregon green; OpuA: osmoprotection uptake system A; rmsd: root mean square deviation; SBP: substrate binding protein; TMD: transmembrane domain or subunit; TR: Texas red.
\end{abstract}




\section{Introduction}

Osmoregulation is of crucial importance for cell viability and survival [1]. Bacillus subtilis (B. subtilis) has to maintain a cell turgor of around 20 bar [2], which is believed to be the driving force of cell expansion. Thus, any change in external osmolarity will induce passive influx (hypoosmotic stress) or efflux (hyperosmotic stress) of water, thereby changing cell turgor $[3,4]$. Hyperosmotic conditions (increase in the extracellular osmolarity) result in the uptake of $\mathrm{K}^{+}$ions as a first line of defense to maintain cell turgor in B. subtilis [5]. However, the accumulation of large concentration of a charged ion is detrimental to many cellular functions such as protein function and/or protein-DNA interaction. Thus, B. subtilis switches to a second line of defense and starts to actively take up or synthesize so-called 'compatible solutes' [6]. In the phyla of Bacteria, these 'compatible solutes' are small molecules such as sugars, polyols, amino acids, quaternary amines, sulfate ester or small peptides [7]. The common denominator of all these compounds is the fact that they are highly soluble and bear no net charge under physiological conditions. As a consequence, compatible solutes counteract the deleterious effect of changes in the extracellular osmolarity by equilibrating concentration gradients across the cellular membrane and maintaining cell turgor [4]. In addition to their well-studied role in maintaining an appropriate level of cell water and turgor, compatible solutes also act as protein stabilizers both in vitro and in vivo. Although the molecular action of compatible solutes as "chemical chaperon" is poorly understood, the common opinion is that these molecules act according to the 'volume exclusion model' [8].

Extensive research in the last two decades has identified five transport systems providing osmoprotection in B. subtilis through the uptake of compatible solutes [6]. In concert, these five importers allow $B$. subtilis to cope with osmotic stress due to the ability to utilize common compatible solutes such as proline, choline, ectoine, glycine betaine or proline betaine. All of the osmoprotectant uptake systems operating in B. subtilis (Opu) belong either to the class of secondary transporters (OpuD and OpuE) or to a subfamily of primary transporters, the ABC- (ATP-binding cassette) transporter family (OpuA, OpuB and $\mathrm{OpuC}$; for a recent review see [9]). These membrane transporters ultimately use the energy of ATP for substrate translocation across biological membranes [10]. Although found in all three kingdoms of life and involved in many vital cellular processes, ATP-dependent transporters share a basic blueprint that is composed of four modules, two nucleotide-binding domains or subunits (NBDs) harboring the ATPase activity and two transmembrane domains or subunits (TMDs) that forms the translocation pathway. Next to the substrate, ABCtransporters can be classified by the directionality of transport, import or export. OpuA belongs to the family of $\mathrm{ABC}$-importers, since it catalyzes the import of glycine betaine and proline betaine from the extracellular media into the bacterial cytosol. Additionally to the NBD (OpuAA) [11] and the TMD (OpuAB) [9], this family always requires another component for functionality, a so-called substrate binding protein (SBP; OpuAC in the case of the OpuA transporter [12]). Recent insights into the molecular function of these ABCtransporters were provided by X-ray crystallography that determined the high-resolution structures of isolated NBDs (for a recent review see [13]) and intact ABC-transporters [1419]. For example, crystal structures of the NBD of the maltose import system (MalK) in the nucleotide-free, ATP- and ADP-bound state have deciphered many of the molecular principles that govern ATP-binding and hydrolysis and resulted in the proposal of a "tweezerlike motion" that accompanies maltose import [20,21].

OpuAA, the NBD of the OpuA transporter, and MalK, the NBD of the maltose permease, share interesting similarities. Both NBDs are not only composed of a catalytic and helical domain that harbors all conserved sequence motifs of these ATPase such as the Walker A and B motifs and the hallmark of ABC-transporters, the C-loop or ABC-signature 
motif [10], but they also contain an accessory domain, which is composed of roughly 150 amino acids and located C-terminal to the canonical NBD (catalytic and helical domain). In the case of MalK, this accessory domain interacts with at least two enzymes, MalT [22] and enzyme IIA [23], thereby regulating the expression of the maltose operon. Furthermore, in the 'tweezer-like motion' model, the accessory domains act as the bottom of tweezers keeping two NBDs together during a catalytic cycle, while the canonical part (catalytic and helical domain) undergoes ATP-induced dimerization remaining monomeric in the nucleotide-free and ADP-bound states.

Biochemical studies have established that OpuAA undergoes a dynamic monomerdimer equilibrium and forms dimers in the nucleotide-free and ATP-bound state, but in striking contrast to MalK becomes monomeric in the ADP-bound state [11]. The closely related OpuA glycine betaine importer in Lactococcus lactis (L. lactis) has been intensively studied in the past by biochemical approaches [24]. Like OpuAA from B. subtilis, the NBD of the L. lactis homologue is composed of a canonical part (catalytic and helical domain) and an accessory domain. Most important was the fact that the accessory domain contains the fingerprint of two CBS domains [25] and is involved in sensing osmotic stress signals that activate the transport function of the L. lactis OpuA system [26].

Here, we have applied Förster resonance energy transfer studies (FRET) to analyze the structure of OpuAA in solution by monitoring and determining the conformational changes taking place during the catalytic cycle of the ATPase. After generating suitable single Cys mutants of OpuAA, static and time-resolved FRET measurements provided a set of distance restrains that were employed to model the overall structures of OpuAA in the nucleotide-free, ATP- and ADP-bound state based on the available crystal structures of MalK [20, 21]. Surprisingly, the three-dimensional positioning of the accessory domains with respect to the canonical part of the NBD was different, while the overall conformational changes during the catalytic cycle were identical to the ones determined previously for other isolated NBDs [20, 27-29]. Thus, our results provide novel insights on the architecture of OpuAA from B. subtilis and suggest that the accessory domains play an important role in the structural and functional regulation of these proteins.

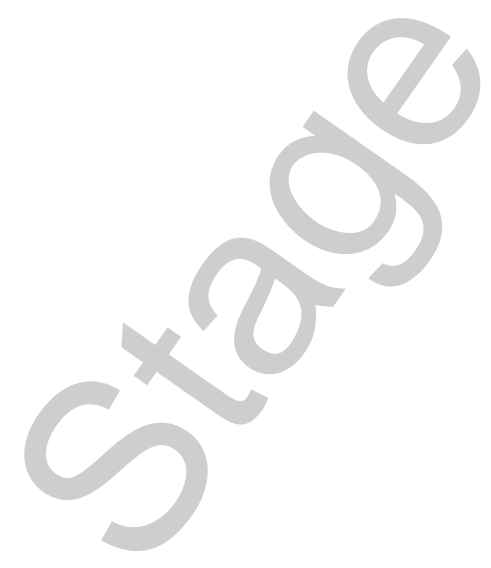




\section{Materials and Methods:}

Cloning, expression and isolation of OpuAA single Cys mutants and the F19W mutant To exchange native amino acids to Cys, 5`and $3^{`}$ fragments were amplified by PCR using pBAD33/OpuAA as template and flanking/mutagenic primer pairs as follows. Ser ${ }^{45}$ Cys: $5^{\prime}$ : CH7/CH29; 3': CH8/CH28. Gly ${ }^{161} \mathrm{Cys}$ : 5': CH7/CH41; 3': CH8/CH40; Phe ${ }^{19}$ Trp: CH47/CH48. CH7: 5'-GGA ATT CCA TAT GAG TGT AGA TGA GAA ACC AAT TA; CH8: 5'-ATA TAT AAA GCT TAT TAG TGA TGG TGA TGG TGA TGT TTC ACC TCC TGT GCA GAA GGA TCT TG; CH28: 5'-ATT AAC CCC AAC GGT ACA TCC GGT TGC TTT CAG; CH29: 5'-CTG AAA GCA ACC GGA TGT ACC GTT GGG GTT AAT; CH40: 5'-ATA TTG GTG TTC AAA ACA TTC CAG TGG AAC GAG, CH41: 5'-CTC GTT GGA CTG GAA TGT TTT GAA CAC CAA TAT, CH47: 5'-ATT CTT TGT TTG TTT CCC CCA AAT CTT AGA GAC-3', CH48: 5'-GTC TCT AAG ATT TGG GGG AAA CAA ACA AAG AAG-3'. Second, the mutated $о p u A A$ gene was amplified using both overlapping fragments as template and the flanking primers $\mathrm{CH}$ 7, $\mathrm{CH} 8$. The PCR products were digested with NdeI/HindIII (New England Biolabs) and cloned into pBAD33. All gene inserts were sequenced according to the ddNTP method and were free of mutations. Mutant proteins were produced in E. coli and purified by IMAC as described [11]. Monomeric OpuAA was isolated by SEC using a Superdex200 HR 26/60 column (GE Healthcare) equilibrated with buffer A $(10 \mathrm{mM} \mathrm{NaP}, 0.1 \mathrm{mM}$ EDTA, $\mathrm{pH} 7.5)$ supplemented with $1 \mathrm{M} \mathrm{NaCl}$. Protein-containing fractions were pooled and stored at $4^{\circ} \mathrm{C}$ until further use.

ATPase activity assays. ATPase activity of monomeric OpuAA was analyzed by an enzymelinked assay as described [11] in $10 \mathrm{mM}$ sodium phosphate $\mathrm{pH} 7.5,1 \mathrm{M} \mathrm{NaCl}$. In brief, absorbance at $340 \mathrm{~nm}$ was monitored at 1 min time intervals over a period of $15 \mathrm{~min}$ at $22 \pm 2$ ${ }^{\circ} \mathrm{C}$. Data points of the whole measuring period or until all NADH was consumed (at least over a period of $7 \mathrm{~min}$ ) were used to calculate linear slopes $\frac{\Delta A}{\Delta t}$ OpuAA . To account for autohydrolysis of ATP, identical assays were performed in the absence of OpuAA. After background subtraction $\left(\frac{\Delta \Delta A}{\Delta \Delta t}=\frac{\Delta A}{\Delta t} t_{\text {OpuAA }}-\frac{\Delta A}{\Delta t}\right.$ buffer $)$ reaction velocities were calculated according to equation 1 .

$v=\frac{\Delta \Delta A}{\Delta \Delta t} \cdot \frac{1}{2590 M^{-1} \cdot c_{0}}$ equation (1)

Here, $\mathrm{v}$ is the reaction velocity, $\frac{\Delta \Delta A}{\Delta \Delta t}$ denote the linear slopes of the time dependent decay of $\mathrm{NADH}$ absorbance at $340 \mathrm{~nm}$ of OpuAA samples after background subtraction and $\mathrm{c}_{0}$ is the final concentration of OpuAA. Reaction velocities represent the average value of two independent measurements.

Fluorophore labeling of OpuAA mutants. For site specific labeling of mutant OpuAA with fluorophore dyes, Cys-specific maleinimide chemistry was used. Typically, OpuAA was passed over a PD-10 colum (GE Healthcare) to remove DTT that was added to prevent disulfide bound formation during storage and mixed at a concentration of $10 \mu \mathrm{M}$ with $25 \mu \mathrm{M}$ dye (donor dye: Oregon Green ${ }^{\circledR} 488$ maleinimide (Molecular Probes), $20 \mathrm{mM}$ in dimethyl formamide; acceptor dye: Texas $\operatorname{Red}^{\circledR} \mathrm{C} 2$ maleinimide (Molecular Probes), $4.1 \mathrm{mM}$ in methanol) and incubated over night at $4^{\circ} \mathrm{C}$. Protein and free dye were separated using a PD-10 column (GE Healthcare) equilibrated with buffer A supplemented with $10 \mathrm{mM} \mathrm{NaCl}$. To isolate the labeled mutants in a quantitative fashion, anion exchange chromatography was 
used to separate labeled and non-labeled OpuAA. After sample-load onto a $1 \mathrm{~mL}$ Q-Sepharose HiTrap column (GE Healthcare) equilibrated with buffer A supplemented with $10 \mathrm{mM} \mathrm{NaCl}$, a 12 column volume gradient $(0.01-2.5 \mathrm{M} \mathrm{NaCl}$ in buffer $\mathrm{A})$ was applied. Elution was followed at $216 \mathrm{~nm}, 491 \mathrm{~nm}$ and $592 \mathrm{~nm}$, respectively.

Labeling efficiency and protein concentration: Protein concentration was determined using the BCA assay following the procedure provided by the manufacturer using BSA as a standard. To determine the efficiency of fluorophore labeling, the molar extinction coefficient of the isolated dyes was used as provided by the manufacture (Molecular Probes). However, not only incomplete labeling was evident but also a blue shift of the emission maximum as well as changes in the molar extinction coefficient (up to 2fold, Figure S2). Thus, an additional purification step to quantitatively separate labeled and non-labeled protein was employed (see above) to circumvent these problems and avoid spectroscopic determination of the labeling efficiency.

Mass Spectrometry: Samples obtained from an anion exchange chromatography (OpuAAOG S45C or OpuAA-TR S45C) or purified OpuAA S45C were desalted over a C18 reversedphase minicolumn (perfect pure C18, Eppendorf, Germany) before mass spectrometric analysis. ESI-MS measurements were performed using a ESI-QqTOF (QSTAR XL, Applied Biosystems, Darmstadt, Germany) equipped with a nanospray ionsource.The ESI-MS spectra were reconstructed using the deconvolution algorithm included in the analyst software (Applied Biosystems, Darmstadt, Germany).

FRET sample preparation. To measure FRET transfer efficiencies within homo-dimeric OpuAA, all donor had to be paired with acceptor in the assembled dimer. Therefore, OpuAATR and OpuAA-OG were mixed in a 9:1 molar ratio. In brief, species were mixed at conditions of dynamic monomer/dimer equilibrium (buffer $\mathrm{A}+100 \mathrm{mM} \mathrm{NaCl}$ ) and concentrated to $30 \mu \mathrm{M}$ to force dimer formation. After incubation over night at $4{ }^{\circ} \mathrm{C}$, samples were applied to a Superdex200 HR 10/30 SEC column (GE Healthcare) previously equilibrated with buffer A supplemented with $1 \mathrm{M} \mathrm{NaCl}$ to isolate the dimeric species. The dimer fraction was used for FRET assay, while the monomer fraction was used only for control experiments. For ADP/ $\mathrm{Mg}^{2+}$ mediated decay the dimer fraction was diluted into buffer A $+100 \mathrm{mM} \mathrm{NaCl}$.

FRET assays. Fluorescence was measured with a Cary-Eclipse spectrometer (Varian) at $\lambda_{\text {exc. }}=492 \mathrm{~nm}, \mathrm{~T}=20 \pm 1^{\circ} \mathrm{C}$ and slid width $=5 \mathrm{~nm}$ in $10 \mathrm{mM}$ sodium phosphate $\mathrm{pH} 7.5,0.1 \mathrm{mM}$ EDTA, $1 \mathrm{M} \mathrm{NaCl}$. In static FRET assays, samples were separately analyzed with emission recording from $500-650 \mathrm{~nm}$ and analyzed according to equation (2) [30]:

$$
E_{T}=\frac{R_{0}^{6}}{R_{0}^{6}+R^{6}}
$$

In time-resolved FRET assays, the sample was chased with $10 \mathrm{mM}$ ADP or ADP/Mg ${ }^{2+} \mathrm{pH} 7.5$ at $\mathrm{t}=0$ to allow dimer dissociation. Fluorescence at time $\mathrm{t}\left(\mathrm{F}_{\mathrm{t}}\right)$ was followed at $519 \mathrm{~nm}$ over a period of $3600 \mathrm{~s}$ at $5 \mathrm{~s}$ intervals and fitted to equation 2. Here, fitting parameters were:

fluorescence at $\mathrm{t}=0\left(\mathrm{~F}_{\mathrm{t}=0}\right)$, fluorescence increase at infinite time $(\Delta \mathrm{F})$ and half-time $(\tau)$.

$$
F_{t}=F_{t=0}+\Delta F \cdot\left(1-e^{-t / \tau}\right)
$$

Amplitudes were used to calculate the $\mathrm{E}_{\mathrm{T}}$-time-course according to equation 3. For $\mathrm{t}=0$ the $\mathrm{E}_{\mathrm{T}}$ value for the original, nucleotide-free state was extrapolated and using the Förster-distance ( $\mathrm{R}_{0}, 51 \AA$ for the OG-TR pair) of this donor/acceptor pair, its distance in this state was calculated.

$$
E_{T}(\hat{t})=1-\frac{F_{t}}{F_{t=0}+\Delta F}=1-\frac{F_{t}}{F_{t=\infty}}=\frac{R_{0}^{6}}{R_{0}^{6}+R(t)^{6}}
$$


Intrinsic tryptophan fluorescence measurements of the F19W mutant of OpuAA. All measurements were performed at $22 \pm 1^{\circ} \mathrm{C}$ on a Carry Eclipse (Varian) employing a slide width of $5 \mathrm{~nm}$. Wild type OpuAA does not contain any tryptophans. The Trp residue engineered at position 19 of OpuAA was exited at $290 \mathrm{~nm}$ and fluorescence was collected at the emission maximum $(350 \mathrm{~nm})$ at a protein concentration of $2 \mu \mathrm{M}$ dimeric OpuAA in 10 $\mathrm{mM}$ sodium phosphate, $\mathrm{pH} 7.5,1 \mathrm{M} \mathrm{NaCl}, 0.1 \mathrm{mM}$ EDTA. The chase was started by adding 1 $\mathrm{mM} A D P$ and $5 \mathrm{mM} \mathrm{Mg}^{2+}$. Data were analyzed using a single exponential decay.

Molecular modeling and structural analysis. Molecular modeling was performed with MODELLER 9v1 [31] employing standard settings. As templates, the crystal structures of MalK in the semi-open, nucleotide-free (pdb entry 1Q1B), the open nucleotide-free (pdb entry 1Q1E), the ATP- (pdb entry 1Q12) and ADP-bound (pdb entry 1AWN) states were used. The quality of the obtained structural models of OpuAA in the corresponding functional states was verified with PROCHECK [32] and compared with the template structure. The initial models were further modified in $\mathrm{O}$ after imposing the FRET-derived distance restrains using sgFRET (www.mpibpc.mpg.de/groups/grubmueller/start/software/fretsg-1.0/fretsg.html). Structural superimpositions were performed using LSQMAN [33] using the indicated motifs as anchor points. BSA were calculated using areaimol of the ccp4 suite. All structural figures were prepared using PyMol (http://www.pymol.org). 


\section{Results:}

Biochemical studies have demonstrated that OpuAA from B. subtilis undergoes a dynamic monomer-dimer equilibrium [11]. Interestingly, this equilibrium depends on the presence or absence of nucleotides. To gain further insights into the underlying conformational changes, we applied FRET techniques of single Cys-mutants of OpuAA in solution. Since the crystal structures of MalK from E. coli are available in the nucleotide-free, ATP- and ADP-bound state [20, 21], they provide a lead structure (homology of $69 \%$ ) for OpuAA homology modeling and would open up the possibility to translate the distance restrains derived from FRET measurements into a three-dimensional model of OpuAA.

A homology model of OpuAA using the crystal structure of MalK in the nucleotidefree state (pdb entry 1Q1B) was generated (see Materials and Methods). As shown in Figure 1A, OpuAA adpots indeed a 'MalK-like' structure [20] composed of a helical (colored in tan) and a catalytic domain (colored in yellow). The catalytic domain of OpuAA contains a 24 amino acid insertion between $\beta$-strand 1 and 2 (highlighted in grey), which is not present in other ABC-domains. However, homology modeling of the accessory domain (represented by a circle in Figure 1A) was not straightforward. In clear contrast to MalK, the primary structure of OpuAA does not contain the finger print of the CUT1 (carbohydrate uptake transporter 1) subfamily represented by a characteristic GI/VRPE consensus sequence [34]. Rather the sequence indicates the presence of two CBS domains (Figure 1B) as evident by a sequence alignment of the accessory domain of OpuAA from B. subtilis with OpuAA from $L$. lactis, inosine-monophosphate dehydrogenase (IMPDH) and the orphan open reading frames from Thermotoga maritimus and Thermophilis acidophilum [25]. These open reading frames were chosen, because crystal structures of the CBS modules are available (see the Figure legends for the corresponding pdb entries). Among the four crystal structures, Thermophilis acidophilum TA0289 had the highest sequence similarity (51\%) and was used for separate homology modeling. The resulting model of the isolated accessory domain of OpuAA is shown in Figure 1C displaying the characteristics of the tandem organization of CBS domains. Since no a priori information of the relative orientation of the accessory and the core NBD domain are available, no attempts were made to include the homology model of the CBS-domain of OpuAA (Figure 1C) into the MalK-derived homology model of OpuAA (Figure 1A).

With the homology model at hand, we selected three residues of OpuAA within the catalytic and helical domains for exchange to cysteine. These residues are highlighted as spheres in Figure 1A. S45 is located in the catalytic domain at the C-terminal end of the OpuAA-specific insertion between $\beta$-strands 1 and 2, G161 N-terminal of the ABC-signature motif. S171, the conserved serine of the ABC-signature motif, was also selected for cysteine mutation to distinguish the composite dimer architecture from the one observed in the MalK crystal structure from $T$. litoralis [35], which shows a different arrangement of the two monomers in the NBD-dimer.

Wild type OpuAA and the three cysteine mutants were overexpressed in E. coli and purified as described in Materials and Methods. Analysis of the two single cysteine mutants (S45C and G161C) under reducing and oxidizing conditions (Figure S1) demonstrated that only negligible intermolecular disulfide bond formation occurred. More important for our conformational analysis was the ATPase activity of the mutant proteins (Table 1). The wild type enzyme hydrolyzed ATP at a rate of $1.1 \pm 0.1 \mathrm{~min}^{-1}$ under the conditions of the assay [11]. In contrast to the S45C and the G161C mutants, which hydrolyzed ATP at comparable rates, the $\mathrm{S} 171 \mathrm{C}$ mutant displayed hardly any turnover $\left(0.08 \pm 0.01 \mathrm{~min}^{-1}\right)$. Due to this result, the S171C mutant was not used for further studies. 
In order to use FRET quantitatively and to extract distance information, it is of importance to obtain a reliable value for the labeling efficiency [36]. The engineered, single cysteine mutants of OpuAA allowed us to employ maleimide chemistry and covalently link the individual fluorophores, Oregon Green (OG) or Texas Red (TR). Surprisingly, labeling efficiencies were rather low (around 50\% for the G161C and around $60 \%$ for the S45C mutant for both dyes). To exclude non-specific labeling due to the prolonged reaction time, wild type OpuAA was treated with the fluorophores under identical conditions. Here, no labeling above background was detected after separating protein and fluorophore (data not shown). Additionally, attachment of the dyes changed their molar extinction coefficient as well as the absorption maximum (12 $\mathrm{nm}$ for TexasRed and $8 \mathrm{~nm}$ for Oregon Green; Figure S2). To account for the low labeling efficiency and the changes in the physical properties of the protein-attached fluorophores, we established a second purification step, anion exchange chromatography, to separate labeled and non-labeled protein (Figure 2). A separation of a labeled and a non-labeled protein species is evident from the absorption properties shown in the representative chromatograms of OpuAA S45C-OG (Figure 2A), OpuAA S45C-TR (Figure 2B), OpuAA G161C-OG (Figure 2C) and OpuAA G161C-TR (Figure 2D).

To further investigate whether the apparent separation was indeed quantitative, we applied electron spray ionization mass spectrometry (ESI-MS) of the two fractions obtained by anion exchange chromatography. ESI-MS analysis of fraction I, which eluted at lower ionic strength, revealed the presence of a single protein species with a molecular weight of $47174 \mathrm{Da}$ (Figure 3A). The calculated average molecular weight of OpuAA S45C including a $\mathrm{C}$-terminal hexahistidine tag and a formylated methionine is $47174.48 \mathrm{Da}$. The second fraction, which eluted at higher ionic strength, revealed the presence of a single protein species of $47657 \mathrm{Da}$ in the case of OpuAA S45C-OG (Figure 3B) and 47921 Da in the case of OpuAA S45C-TR (Figure 3C). These mass differences correspond within experimental error of the MS measurements to the molecular weights of the individual fluorophores (differences of $483 \mathrm{Da}$ for the sodium adduct of OG (expected 486.35 Da) and $747 \mathrm{Da}$ for the sodium adduct of TR (expected $751.83 \mathrm{Da})$ ).

A detailed analysis of the monomer-dimer equilibrium of OpuAA [11], has provided a toolbox to generate monomers or dimers of OpuAA depending on the protein concentration and ionic strength. This allowed us to prepare OpuAA-OG/OpuAA-TR pairs at a molar ratio of 1:9, which ensures that all (> 95\%) OG-labeled OpuAA molecules are indeed in an OG/TR dimer. On the other hand, a high molar excess of acceptor might result in an apparent increase in transfer efficiency due to radiation-dependent processes such as acceptor self-absorption. However, these processes dependent solely on the concentration of the acceptor and a proper choice will eliminate this obstacle. Therefore, we measured the fluorescence of a $50 \mathrm{nM}$ sample of OpuAA-OG (donor) in the presence or absence of a 9fold molar excess of TRconjugated OpuAA (spectra not shown) under conditions that abolish FRET. Comparison of the two spectra clearly showed that marginally self-absorption of TR occurred. Consequently, we used a fixed donor concentration of $50 \mathrm{nM}$ in all subsequent experiments.

A representative set of static FRET experiments is shown in Figure 4 for the OpuAA S45C-OG/OpuAA S45C-TR dimer in the nucleotide-free (Figure 4A), the ATP-bound (Figure 4B) and the ADP-bound state (Figure 4C). The dashed black lines show the sum of two control experiments, OpuAA S45C-OG and wild type OpuAA (molar ration of 1:9) and wild type OpuAA and OpuAA S45C-TR (molar ratio of 1:9), while the solid lines show the fluorescence spectrum of a OpuAA S45C-OG/OpuAA S45C-TR mixture (molar ration of 1:9). The insets show the corresponding difference spectra. As evident from these experiments, FRET can be detected for the nucleotide-free and the ATP-bound state of 
dimeric OpuAA, but not for the ADP-bound state. The FRET efficiencies were calculated to $34 \%$ and $39 \%$ for the nucleotide-free and ATP-bound state according to equation (2). A summary of the FRET efficiencies determined for this and the other combinations is given in Table 2.

Distance determinations through static FRET experiments require an accurate knowledge of the protein concentration. In the studies presented here, concentration determination was complicated by the fact that the molar extinction coefficients of the dyes changed upon covalent attachment to the protein (see Figure S2). We determined protein concentrations as outline in Materials and Methods and incorporated the changed molar extinction coefficients determined under the experimental conditions, but any error in the determination would drastically affect the FRET distances extracted from those measurements. Thus, we also thought of establishing an experimental approach that is independent of a prior, exact knowledge of protein concentration. Here, time-resolved changes in donor fluorescence of monomeric or dimeric OpuAA G161C-OG/OpuAA G161CTR, dimeric OpuAA G161C-OG/OpuAA S45C-TR and dimeric OpuAA S45C-OG/OpuAA S45C-TR (Figure 5A) provided a tool to counter check the distance information of the static FRET experiments and to validate these results. Nucleotide-free samples of the indicated combinations were incubated with $\mathrm{ADP} / \mathrm{Mg}^{2+}$ and fluorescence was measured over the indicated time frame. The observed fluorescence increased over time in a strictly monoexponential fashion for all dimeric probes, while it remained constant for the monomeric probe. Thus, the increase in donor fluorescence corresponds to dimer decay and the decay reaction was evaluated according to equation (3). This analysis provided the amplitudes and half times of dimer decay and could be used to calculate the transfer efficiency for the individual combinations according to equation (4) by extrapolation of the data to $t=0$ (Figure 5B). This extrapolation allowed us to extract the transfer efficiencies without prior knowledge of protein concentration or the exact starting time point of the experiment. A summary of the calculated values is given in Table 3 . This demonstrates the agreement between transfer efficiencies determined by static and time-resolved FRET.

Additional support for the ADP-induced dimer decay comes from independent experiments using the F19W mutant of OpuAA (Figure 6). F19, which based on sequence comparisons is interacting with bound nucleotide via $\pi-\pi$ stacking, was mutated to Trp to use intrinsic tryptophan fluorescence quenching to monitor nucleotide association and dissociation similar to the approach employed for the isolated NBD of HlyB [37]. Kinetic parameters of this mutant are summarized in Table 1. As shown in Figure 6, chasing of dimeric, nucleotide-free OpuAA with ADP, resulted in a quenching of the detected Trp fluorescence. As analyzed for the monomeric form of OpuAA, the observed fluorescence reduction is due to nucleotide binding and $\pi-\pi$ interaction of the adenine moiety of ADP or ATP and W19 (Figure S3). However, the determined half time of fluorescence decay (18 \pm 4 $\mathrm{min}$ ) is similiar with the half times determined for the time-resolved fluorescence experiments within experimental error (Table 3).

Based on the FRET-derived distance information for the two homo-pairs (S45C/S45C and $\mathrm{G} 161 \mathrm{C} / \mathrm{G} 161 \mathrm{C})$ and the hetero-pair (S45C/G161C) in the nucleotide-free and ATP-bound state (Tables 2 and 3), a molecular model was created using FRETsg (www.mpibpc.mpg.de/groups/grubmueller/start/software/fretsg-1.0/fretsg.html) as anchor points for a structural alignment in $\mathrm{O}$ [38]. While the $\mathrm{C} \alpha-\mathrm{C} \alpha$ distances obtained from the FRET studies of the homo-pairs $(56.9 \AA(45-45)$ and $51.3 \AA(161-161))$ agreed with the open conformation of MalK (59.9 $\AA$ and $51.9 \AA$, respectively) [20], the distance of the $\mathrm{S} 45 \mathrm{C} / \mathrm{G} 161 \mathrm{C}$ was roughly twice the distance measured in MalK (52.1 $\AA$ versus $22.3 \AA$ ). Thus, the open, nucleotide-free dimer of MalK was adjusted accordingly. Here, one monomer 
was kept fixed while the other was treated as a rigid-body. The resulting model for OpuAA satisfying all FRET-derived distance restrains is shown in Figure 7A. The canonical part of each NBD monomer, composed of the catalytic and helical subdomain, is shown in yellow and light orange, respectively, and the accessory domains in grey. The Walker A and $\mathrm{ABC}$ signature motifs are highlighted in blue and red, respectively, while the $\mathrm{C} \alpha$ trace of the amino acids, which were mutated to cysteine and labeled with the corresponding fluorophores, is colored in green (position 45) and dark grey (position 161). Thin, dashed lines indicate the distances between the labeled amino acids, which were calculated from the FRETmeasurements. Interestingly, the closest $\mathrm{C} \alpha-\mathrm{C} \alpha$ distance between opposing Walker $\mathrm{A}$ and $\mathrm{ABC}$-signature motifs are 17 and $20 \AA$, respectively. These values agree with the distance determined for the corresponding $\mathrm{C} \alpha$-atoms of nucleotide-free, open form of MalK (17 $\AA$ ) from $E$. coli [20]. Thus, it appears that the overall structure of the canonical part of the NBD is identical between OpuAA and MalK. However, the accessory domains are in a different orientation than expected from the MalK structure. As a consequence severe clashes occur between the canonical and accessory part of opposing OpuAA monomers (Figure 7A) indicating that the accessory domain has to adopt a different orientation. Furthermore, the Dloop motifs (SALD in OpuAA and SNLD in MalK) of opposing monomers are in close contact $(\mathrm{C} \alpha-\mathrm{C} \alpha$ distance of $7 \AA)$. As evident in Figure $7 \mathrm{~B}$, structural superimposition reveals that one monomer of the OpuAA model aligns very well with a monomer of the nucleotidefree, open structure of MalK (rmsd of $1 \AA$ over $381 \mathrm{C} \alpha$ atoms). The two monomers of MalK are colored in magenta and cyan and the Walker $\mathrm{A}$ and $\mathrm{ABC}$-signature motifs in blue and red, respectively. In clear contrast, the second monomer displayed a translational movement of 9.5 $\AA$ as calculated for the conserved Lys residue of the Walker A motifs, which are shown as blue ribbons ( $\mathrm{Lys}^{42}$ in MalK and Lys ${ }^{72}$ in OpuAA). As a consequence, the accessory domain of the second monomer of the OpuAA model (light grey) is displaced compared with MalK structure (light magenta). A structural superimposition of the OpuAA model and the nucleotide-free, semi-open form of MalK [20] revealed, one monomer imposes slightly less accurate with OpuAA (rmsd of $1.9 \AA$ over $336 \mathrm{C} \alpha$ atoms; data not shown). The $\mathrm{C} \alpha-\mathrm{C} \alpha$ distance of opposing Walker $\mathrm{A}$ and $\mathrm{ABC}$-signature motifs is also shorter $(13 \AA)$ than in the FRET-derived model of OpuAA (17 and $20 \AA$, respectively).

Following the same strategy as outline for the nucleotide-free state of OpuAA, a model was derived for the ATP-bound dimeric state (Figure 8A) that satisfied the distance restrains derived from FRET measurements in solution (Table 2). Here, the $\mathrm{C} \alpha-\mathrm{C} \alpha$ distance between opposing Walker A and ABC-signature motifs shortens to $13 \AA$ compared to the model of the nucleotide-free state. Furthermore, a superposition of the nucleotide-free and the ATP-bound state of the OpuAA model (Figure 8B) reveal that the canonical part of the NBD, colored cyan and magenta, undergoes an induced fit upon ATP-binding, i.e. a rigid-body motion of the helical domain towards the catalytic domain.

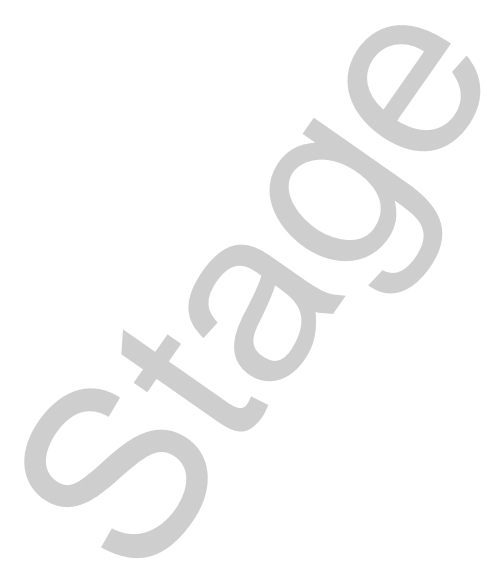




\section{Discussion:}

The large body of biochemical data [39] and the crystal structures of MalK [20, 21], the isolated NBD of the maltose transporter, combined with the recently published crystal structure of the full-length transporter [18], has provided a molecular picture of how substrate import is catalyzed by this $\mathrm{ABC}$ importer. Thus, a "tweezers-like" motion was proposed for MalK. Here, the additional domains of MalK form the bottom of the tweezers keeping two MalK monomers together during the catalytic cycle, while the catalytic and helical domains of the MalK monomers undergo nucleotide-dependent association and dissociation reactions. A similar monomer-dimer equilibrium was observed for OpuAA from B. subtilis [11]. However, in contrast to MalK, dimeric OpuAA was only observed in the absence of nucleotides or the presence of ATP, while it remained monomeric in the presence of ADP. Moreover, dimeric OpuAA could be converted into the monomeric form in the presence of excess ADP. Such an ADP-induced monomerization of OpuAA but not of MalK might be due to differences in the accessory domains. In contrast to MalK, the primary structure of OpuAA indicates the presence of two CBS domains arranged in tandem (Figure 1). CBS domains are found for example in voltage-gated chloride channels [40] or IMP dehydrogenases [41] and are thought to bind small molecules containing an adenosyl moiety such as AMP thereby regulating enzyme activity $[42,43]$. However, ATPase assays of OpuAA in the presence of AMP had no effect on activity and the stoichiometry of ATP (data not shown and [11]). This suggests that the CBS-domains of OpuAA do not interact with AMP in a functional relevant manner. Nevertheless, the core domain of MalK (catalytic and helical domain) is of sufficient homology (36\% identity and $69 \%$ homology) to OpuAA to allow homology modeling (Figure 1), while a homology model of the accessory domain of OpuAA was constructed based on its closest, structurally known homologue, TA0289 (Figure 1C). Based on this model, three amino acids were chosen and converted into cysteines (S45C, G161C and S171C) for subsequent fluorophores labeling.

Wild type and the three mutant proteins could be overexpressed and purified to homogeneity (Figure S1). As summarized in Table 1, turnover rates for the wild type enzyme and the $\mathrm{S} 45 \mathrm{C}$ and $\mathrm{G} 161 \mathrm{C}$ mutants were identical within experimental error. Also the $\mathrm{K}_{\mathrm{M}}$ values for the wild type enzyme and the $\mathrm{S} 45 \mathrm{C}$ were identical, while the $\mathrm{K}_{\mathrm{M}}$ value for the G161C mutant increased by a factor of 5. The result is somewhat surprising since G161 is a surface exposed residue and the structural model provides no explanation for this result. Since all subsequent experiments were performed under ATP saturating conditions, these changes in $\mathrm{K}_{\mathrm{M}}$ do not influence the results. In clear contrast the S171C showed no ATPase activity and was not used in the following analysis. Comparing this activity to the in vivo activity is not possible, because no data on the number of transporter per cell exist [44]. However, assuming an activity typical for ATP-transporters (around $1 \mathrm{ATP} / \mathrm{s}$ ), indicates that the activity of the isolated NBD in vitro is 60 times lower than expected. But already in detergent solution, a 10 fold increase of the ATPase activity was observed in the presence of the TMD and the substrate-loaded SBP [9]. This clearly suggests that a properly assembled transporter requires higher activity than displayed by the isolated NBD. However, the results of the re-assembly studies in detergent solution [9] support the notion that the isolated NBD is properly folded and adopts a transport-competent state that can be stimulated in a SBP- and substratedependent manner.

The S45C and S161C were labeled employing two, thiol-specific maleinimide fluorophores, OG and TR. The use of TR and OG, an efficient FRET pair [30] with a Förster radius of $51 \AA$, should in principle allow us to extract distance information in the different functional states of the isolated NBD, i.e. nucleotide-free, ATP- and ADP-bound. However, labeling efficiencies were at best $60 \%$, a result that cannot be explained based on the 
homology model, because the two residues chosen are surface exposed. Instead of trying to optimize labeling efficiency, we developed an additional purification step to separate labeled and non-labeled species (Figure 2). Although the ion exchange chromatogram indicated quantitative separation, we further analyzed the identity and homogeneity of the two fractions obtained after ion exchange chromatography by ESI-MS (Figure 3). Importantly, the amount of non-labeled OpuAA as indicated by an arrow in Figures 4B and 4C was below $2 \%$ as calculated from the peak areas the ESI-MS spectra of OpuAA S45C-OG and OpuAA S45CTR, respectively. These two independent sets of experimental evidence demonstrate a quantitative separation of labeled and non-labeled OpuAA, which is a prerequisite for subsequent, quantitative FRET measurements.

A process to assemble and de-assemble homodimers of OpuAA had already been described in detail [11] and was readily applied to the labeled single cysteine mutants (see Results). However, FRET efficiency strongly depends on a complete donor-acceptor pairing [30]. Statistic mixing of a 1:1 mixture of OG- and TR-labeled OpuAA would in theory result in the formation of $25 \%$ dimers composed of donors only (OG/OG), $25 \%$ dimers composed of acceptors only (TR/TR) and 50\% composed of donor and acceptor (OG/TR). Although such statistical mixing has been verified experimentally for other isolated ABC-NBDs [37, 45, 46] and additionally a mathematical treatment to correct incomplete assembly is possible in principle, the dynamic monomer-dimer equilibrium of OpuAA would further complicate the evaluation. Therefore, we decide to use a molar ratio of donor: acceptor of 1:9 to ensure quantitative "pairing" of OpuAA-OG with OpuAA-TR. Under these conditions, FRET could be observed for the 45-45, 45-161 and 161-161 pairs in the nucleotide-free and ATP-bound state, but not in the ADP-bound state (Figure 4). Transfer efficiencies are summarized in Table 2. In light of the biochemical characterization of the isolated NBD of the OpuA transporter [11], these results demonstrate that a dimer is present in the nucleotide-free and ATP-bound state, respectively, while the protein is monomeric in the ADP-bound state. However, one has to realize that a determination of transfer efficiencies relies on an accurate protein concentration determination. Since the molar extinction coefficient of the dyes changed upon coupling (Figure S2), protein concentration determination might become unreliable. To overcome, this problem, we also performed time-resolved FRET experiments (Figure 5). Here, the decay in FRET signal of nucleotide-free or ATP-bound dimeric OpuAA samples was measured over time in the presence of $10 \mathrm{mM} \mathrm{ADP}$, which induces dimer dissociation. From the determined amplitudes and the half time of dimer decay, transfer efficiencies could be calculated in a protein concentration independent way (Table 3). The agreement between these values and the values determined from the static measurements (Table 2) suggests that the distances, which can be derived from the transfer efficiencies, can be used as distance restrains for modeling the dimeric state of OpuAA.

Similar to the HlyB system, the introduction of a Trp at position 19 of OpuAA lowered the catalytic efficiency of the enzyme twofold (Table 1). However, in our assays saturating ligand concentrations were used and more important, hydrolysis was prevented by adding EDTA to the buffer so that the enzymatic activity is not as important and the twofold reduction can be tolerated. Thus, the chase of the nucleotide-free dimer of OpuAA F19W with $\mathrm{ADP}$ or $\mathrm{ADP} / \mathrm{Mg}^{2+}$ displays the same result, as the ADP-induced dissociation of the OpuAA dimer detected by fluorescence, but FRET experiments report changes in distance, i.e. dimer decay directly, the F19W mutant uses nucleotide association/dissociation as read-out. However, one has to keep in mind that the $\mathrm{k}_{\text {cat }}$ value of ATPase activity under these conditions is roughly $1 \mathrm{~min}^{-1}$ (Table 1 and [11]), while the half time of dimer decay was between $12.9 \pm 0.1$ and $22 \pm 0.3 \mathrm{~min}$. Stopped-flow experiments employing changes of the intrinisc tryptophan signal (data not shown), revealed that nucleotide association was faster than the time resolution of the experiment (around $1 \mathrm{~ms}$ ). Thus, ATPase activity is either 
limited by hydrolysis as suggested for the HlyB-NBD [28] or the dissociation of the dimer as suggested for MJ1276 [27]. The current model of ATP-hydrolysis within ABC-domains assumes that dimer decay occurs every time the two ATPs bound to the composite dimer are hydrolyzed. Such a scenario is hard to imagine for OpuAA, because $\mathrm{k}_{\mathrm{cat}}$ of $1 \mathrm{~min}^{-1}$ and the half time of dimer decay (between 12 and $22 \mathrm{~min}$ ) are not compatible with this model. However, Gadsby and coworkers [47] have demonstrated that in CFTR, repetitive cycles of ATP hydrolysis can occur before the dimer decays. Clearly, in CFTR the NBD composite sites are non-equal, because the degeneration of certain residues critical for catalysis generates one ATP binding site with no or only little ATP-activity, while the other site contains the canonical residues and is capable of hydrolyzing ATP. Although OpuAA does not contain asymmetric ATP-binding site, it is tempting to speculate that the 10- to 20 -fold difference between ATP turnover and dimer decay is due to a similar phenomena. ATP hydrolysis and dimer decay are not strictly coupled and repetitive cycles can occur in both binding sites before the dimer dissociates. Dimer decay on the other hand would finally terminate ATPase activity. That would also suggest that a single ATP hydrolysis event and the overall termination of activity (dimer decay) might have different rate limiting steps. However, this hypothesis requires further verification and although appealing, the differences between CFTR and OpuAA in sequence and the resulting functional consequences are obvious and also here further experimental analysis is necessary before the discrepancy between turnover and dimer decay can be resolved.

Based on the distance restrains derived from the static and time-resolved FRET measurements (Table 2 and 3), the dimeric homology models of OpuAA (nucleotide-free and ATP-bound) that were based on the dimeric crystal structures of MalK, were modified to satisfy the restrains (Figure 7 and 9). As shown, in Figure 7A and 9A, the FRET-derived distances can only be incorporated into the homology models, if the accessory domains are not in close contact in OpuAA as they are in MalK. Furthermore, the catalytic and helical domains in the second monomer are translationally shifted by $9 \AA$ if comparing MalK and OpuAA (Figure 7B). This excludes that a simple rotation of the linker between core NBD and CBS containing accessory domain is sufficient to generate a 'MalK-like' arrangement. However, one has to stress that the accessory domain of the OpuAA model shown here, contains the MalK-fold, while sequence analysis clearly revealed the presence of CBS domains in OpuAA that adopt a fold similar to the one shown in Figure 1C. The low structural homology between these two folds prohibited any further homology modeling. Analysis of the buried surface area (BSA) of the OpuAA model revealed a value of $130 \AA^{2}$ compared to $1900 \AA^{2}$ in the ATP-bound state of MalK [20]. Clearly, the calculated value of the BSA is too small to explain the dimeric nature of nucleotide-free OpuAA in solution [11]. However, the accessory domains, as shown in Figure 7A, do not have any contact, so that the only stabilization is provided by interactions of the canonical part (helical and catalytic domain) of OpuAA. Thus, the FRET-derived model strongly suggests that the CBScontaining accessory domains stabilize the dimeric nature of this ATPase subunit, but that the relative orientation of canonical NBD and accessory domain is different in OpuAA and MalK. This finding is similar to he situation observed in the crystal structure of CysA [48], the NBD of a putative sulfate import system. Here, the orientation of the accessory domain in one of the two dimers is also different to MalK and suggests that the relative three-dimensional orientation of the core NBD and the accessory domain is different in different NBDs and might be a consequence of functional requirements imposed on the transporter (nature of substrate, interacting molecules/proteins, etc.). On the other hand, a rigid-body inward motion is obseryed for the helical domain of the ATP-bound model compared with the nucleotidefree state (Figure 8B). This rigid-body motion of induced fit upon ATP-binding has been first proposed for MJ1276 [49] and subsequently verified in the crystal structures of other isolated NBDs in the ATP-bound state [20,27-29]. Here, the inward motion ranged from $16^{\circ}$ to $25^{\circ}$. 
In our model, the helical domain of the nucleotide-free state (colored yellow and light orange) undergoes an inward motion of roughly $17^{\circ}$. This demonstrates that the basic motion in the core NBD is also preserved in OpuAA.

In summary, while the orientation of the accessory domain is not identical in OpuAA and MalK, the basic principles of structural changes occurring within the helical domain upon ATP-binding, that have been elaborated for many systems, are also conserved in OpuAA. It is also tempting to speculate that the OpuAA dimer adopts the open conformation of MalK in solution in the absence of nucleotides and not the semi-open state but with a different arrangement of the accessory domain that very likely stabilizes the dimer and also performs a tweezers-like function as it was proposed for MalK [20]. Further studies such as additional FRET or Cys-based cross-linking experiments are necessary to determine the exact orientation of the CBS-domain to each other, but a definite answer will come available only upon determining the three-dimensional crystal structure of OpuAA in different functional states. However, in light of the proposed role of this module in regulating transport function, it is important to realize that not only the primary structure but also the quaternary arrangement is important for in vivo function. 


\section{References}

1 Wood, J. M., Bremer, E., Csonka, L. N., Kraemer, R., Poolman, B., van der Heide, T. and Smith, L. T. (2001) Osmosensing and osmoregulatory compatible solute accumulation by bacteria. Comp. Biochem. Physiol. A Mol. Integr. Physiol. 130, 437-460

2 Whatmore, A. M. and Reed, R. H. (1990) Determination of turgor pressure in Bacillus subtilis: a possible role for $\mathrm{K}^{+}$in turgor regulation. J. Gen. Microbiol. 136 ( Pt 12), 2521-2526

3 Miller, K. J. and Wood, J. M. (1996) Osmoadaptation by rhizosphere bacteria. Annu. Rev. Microbiol. 50, 101-136

4 Wood, J. M. (1999) Osmosensing by bacteria: signals and membrane-based sensors. Microbiol. Mol. Biol. Rev. 63, 230-262.

5 Ventosa, A., Nieto, J. J. and Oren, A. (1998) Biology of moderately halophilic aerobic bacteria. Microbiol. Mol. Biol. Rev. 62, 504-544

$6 \quad$ Kempf, B. and Bremer, E. (1998) Uptake and synthesis of compatible solutes as microbial stress responses to high-osmolality environments. Arch. Microbio1, 170, 319-330

7 Welsh, D. T. (2000) Ecological significance of compatible solute accumulation by micro-organisms: from single cells to global climate. FEMS Microbiol. Rev. 24, 263-290.

8 Minton, A. P. (2001) The influence of macromolecular crowding and macromolecular confinement on biochemical reactions in physiological media. J. Biol. Chem. 276, 1057710580

9 Horn, C., Bremer, E. and Schmitt, L. (2005) Functional overexpression and in vitro assembly of OpuA, an osmotically regulated ABC-transporter from Bacillus subtilis. FEBS Lett. 579, 5765-5768

10 Schmitt, L. and Tampé, R. (2002) Structure and mechanism of ABC-transporters. Cur. Opin. Struc. Biol. 12, 754-760

11 Horn, C., Bremer, E. and Schmitt, L. (2003) Nucleotide dependent monomer/dimer equilibrium of OpuAA, the nucleotide-binding protein of the osmotically regulated ABC transporter OpuA from Bacillus subtilis. J. Mol. Biol. 334, 403-419

12 Horn, C., Sohn-Bosser, L., Breed, J., Welte, W., Schmitt, L. and Bremer, E. (2006) Molecular determinants for substrate specificity of the ligand-binding protein OpuAC from Bacillus subtilis for the compatible solutes glycine betaine and proline betaine. J. Mol. Biol. 357, 592-606

13 Oswald, C., Holland, I. B. and Schmitt, L. (2006) The motor domains of ABCtransporters / What can structures tell us? Naunyn Schmiedebergs Arch. Pharmacol. 372, 385399

14 Dawson, R. J. and Locher, K. P. (2006) Structure of a bacterial multidrug ABC transporter. Nature. 443, 180-185

15 Locher, K. P., Lee, A. T. and Rees, D. C. (2002) The E. coli BtuCD structure: a framework for $\mathrm{ABC}$ transporter architecture and mechanism. Science. 296, 1091-1098

16 Pinkett, H. W., Lee, A. T., Lum, P., Locher, K. P. and Rees, D. C. (2007) An inwardfacing conformation of a putative metal-chelate-type ABC transporter. Science. 315, 373-377 17 Hollenstein, K., Frei, D. C. and Locher, K. P. (2007) Structure of an ABC transporter in complex with its binding protein. Nature. 446, 213-216

18 Oldham, M. L., Khare, D., Quiocho, F. A., Davidson, A. L. and Chen, J. (2007)

Crystal structure of a catalytic intermediate of the maltose transporter. Nature. 450, 515-521

19 Hvorup, R. N., Goetz, B. A., Niederer, M., Hollenstein, K., Perozo, E. and Locher, K. P. (2007) Asymmetry in the structure of the ABC transporter-binding protein complex BtuCD-BtuF. Science. 317, 1387-1390

20 Chen, J., Lu, G., Lin, J., Davidson, A. L. and Quiocho, F. A. (2003) A tweezers-like motion of the ATP-binding cassette dimer in an ABC transport cycle. Mol. Cell. 12, 651-661 
21 Lu, G., Westbrooks, J. M., Davidson, A. L. and Chen, J. (2005) ATP hydrolysis is required to reset the ATP-binding cassette dimer into the resting-state conformation. Proc. Nat. Acad. Sci. USA. 102, 17969-17974

22 Panagiotidis, C. H., Boos, W. and Shuman, H. A. (1998) The ATP-binding cassette subunit of the maltose transporter MalK antagonizes MalT, the activator of the Escherichia coli mal regulon. Mol. Microbiol. 30, 535-546

23 Bluschke, B., Volkmer-Engert, R. and Schneider, E. (2006) Topography of the surface of the signal-transducing protein EIIA(Glc) that interacts with the MalK subunits of the maltose ATP-binding cassette transporter (MalFGK2) of Salmonella typhimurium. J. Biol. Chem. 281, 12833-12840

24 van der Heide, T. and Poolman, B. (2000) Glycine betaine transport in Lactococcus lactis is osmotically regulated at the level of expression and translocation activity. $\mathrm{J}$.

Bacteriol. 182, 203-206

25 Bateman, A. (1997) The structure of a domain common to archaebacteria and the homocystinuria disease protein. Trends Biochem. Sci. 22, 12-13

26 Biemans-Oldehinkel, E., Mahmood, N. A. and Poolman, B. (2006) A sensor for intracellular ionic strength. Proc. Natl. Acad. Sci. USA. 103, 10624-10629

27 Smith, P. C., Karpowich, N., Millen, L., Moody, J. E., Rosen, J., Thomas, P. J. and Hunt, J. F. (2002) ATP binding to the motor domain from an ABC transporter drives formation of a nucleotide sandwich dimer. Mol. Cell. 10, 139-149

28 Zaitseva, J., Jenewein, S., Jumpertz, T., Holland, I. B. and Schmitt, L. (2005) H662 is the linchpin of ATP hydrolysis in the nucleotide-binding domain of the ABC transporter HlyB. EMBO J. 24, 1901-1910

29 Zaitseva, J., Oswald, C., Jumpertz, T., Jenewein, S., Wiedenmann, A., Holland, I. B. and Schmitt, L. (2006) A structural analysis of asymmetry required for catalytic activity of an ABC-ATPase domain dimer. EMBO J. 25, 3432-3443

30 Lakowicz, J. R. (1999) Principles of fluoresccence spectroscopy. Kluwer Academic Publishers, New York

31 Marti-Renom, M. A., Stuart, A. C., Fiser, A., Sanchez, R., Melo, F. and Sali, A. (2000) Comparative protein structure modeling of genes and genomes. Annu. Rev. Biophys. Biomol. Struct. 29, 291-325

32 Laskowski, R. A., MacArthur, M. W., Moss, D. S. and Thornton, J. M. (1993)

PROCHECK: a program to check the stereochemical quality of protein structures. J. Appl. Crystallogr. 26, 283-291

33 Kleywegt, G. J. (1996) Use of non-crystallographic symmetry in protein structure refinement. Acta Crystallogr. D 52, 842-857

34 Schneider, E. (2001) ABC transporters catalyzing carbohydrate uptake. Res. Microbiol. 152, 303-310

35 Diederichs, K., Diez, J., Greller, G., Muller, C., Breed, J., Schnell, C., Vonrhein, C., Boos, W. and Welte, W. (2000) Crystal structure of MalK, the ATPase subunit of the trehalose/maltose ABC transporter of the archaeon Thermococcus litoralis. EMBO J. 19, 5951-5961

36 Berney, C. and Danuser, G. (2003) FRET or no FRET: a quantitative comparison. Biophys. J. 84, 3992-4010

37 Zaitseva, J., Jenewein, S., Wiedenmann, A., Benabdelhak, H., Holland, I. B. and

Schmitt, L. (2005) Functional characterization and ATP induced dimerization of the isolated ABC-domain of the haemolysin B transporter. Biochemistry. 44, 9680-9690

38 Jones, T. A., Zou, J. Y., Cowan, S. W. and Kjeldgaard. (1991) Improved methods for binding protein models in electron density maps and the location of errors in these models. Acta Crystallogr A. 47, 110-119

39 Davidson, A. L. and Chen, J. (2004) ATP-binding cassette transporters in bacteria. Annu. Rev. Biochem. 73, 241-268 
40 Meyer, S. and Dutzler, R. (2006) Crystal structure of the cytoplasmic domain of the chloride channel ClC-0. Structure. 14, 299-307

41 Zhang, R., Evans, G., Rotella, F. J., Westbrook, E. M., Beno, D., Huberman, E., Joachimiak, A. and Collart, F. R. (1999) Characteristics and crystal structure of bacterial inosine-5'-monophosphate dehydrogenase. Biochemistry. 38, 4691-4700

42 Bennetts, B., Rychkov, G. Y., Ng, H. L., Morton, C. J., Stapleton, D., Parker, M. W. and Cromer, B. A. (2005) Cytoplasmic ATP-sensing domains regulate gating of skeletal muscle ClC-1 chloride channels. J. Biol. Chem. 280, 32452-32458

43 Scott, J. W., Hawley, S. A., Green, K. A., Anis, M., Stewart, G., Scullion, G. A., Norman, D. G. and Hardie, D. G. (2004) CBS domains form energy-sensing modules whose binding of adenosine ligands is disrupted by disease mutations. J. Clin. Invest. 113, 274-284 44 Kempf, B. and Bremer, E. (1995) OpuA, an osmotically regulated binding proteindependent transport system for the osmoprotectant glycine betaine in Bacillus subtilis. J. Biol. Chem. 270, 16701-16713

45 Janas, E., Hofacker, M., Chen, M., Gompf, S., van der Does, C. and Tampe, R. (2003) The ATP hydrolysis cycle of the nucleotide-binding domain of the mitochondrial ATPbinding cassette transporter Mdl1p. J. Biol. Chem. 278, 26862-26869

46 Nikaido, K. and Ames, G. F. (1999) One intact ATP-binding subunit is sufficient to support ATP hydrolysis and translocation in an ABC transporter, the histidine permease. J. Biol. Chem. 274, 26727-26735

47 Vergani, P., Lockless, S. W., Nairn, A. C. and Gadsby, D. C. (2005) CFTR channel opening by ATP-driven tight dimerization of its nucleotide-binding domains. Nature. 433, 876-880

48 Scheffel, F., Demmer, U., Warkentin, E., Hulsmann, A., Schneider, E. and Ermler, U. (2005) Structure of the ATPase subunit CysA of the putative sulfate ATP-binding cassette (ABC) transporter from Alicyclobacillus acidocaldarius. FEBS Lett. 579, 2953-2958 49 Karpowich, N., Martsinkevich, O., Millen, L., Yuan, Y. R., Dai, P. L., MacVey, K., Thomas, P. J. and Hunt, J. F. (2001) Crystal structures of the MJ1267 ATP binding cassette reveal an induced-fit effect at the ATPase active site of an ABC transporter. Structure. 9, 571586 


\section{Acknowledgements:}

We thank Suman Lata, Jacob Piehler, and all members of the Institute of Biochemistry for the stimulating discussions and Robert Tampé for constant support and encouragement. We are grateful to Filip Oesterhelt, Claus Seidel and Sander Smits for many insightful discussions concerning the FRET experiments and their interpretations. This work was supported by the DFG (special priority program 1070, grants BR 796/51 to E.B. and Schm1279/4-1 to L.S.). 


\section{Table Legends:}

Table 1: Kinetic parameters of monomeric OpuAA and the mutants used in this study. ATPase assays were performed in $10 \mathrm{mM}$ sodium phosphate $\mathrm{pH} 7.5,1 \mathrm{M} \mathrm{NaCl}$ as described in Material and Methods. Values represent the average of two independent measurements with the standard deviation reported as errors.

Table 2:

Summary of the static and time-resolved FRET experiments. The dimeric species of the indicated single cysteine mutants of OpuAA of the nucleotide-free and ATP-bound state and the monomeric species of the indicated single cysteine mutants of OpuAA in the ADP-bound state were analyzed as described in Materials and Methods in the absence of $\mathrm{Mg}^{2+}$. The determined transfer efficiencies at $519 \mathrm{~nm}$ (donor emission maximum) or the tatic FRET experiments were translated in distances according to the Förster equation assuming a Förster radius of the OG/TR pair of $51 \AA$ [30]. In the case of the time-resolved experiments, amplitudes, half-times and half times were calculated from the ADP/ $\mathrm{Mg}^{2+}$-induced decay of the dimeric forms of labeled OpuAA mutants as described in Materials and Methods. 
Table 1

\begin{tabular}{|l|l|l|l|}
\hline Protein & $\mathrm{K}_{\mathrm{M}}[\mathrm{mM}]$ & $\mathrm{k}_{\text {cat }}[\mathrm{ATP} / \mathrm{min}]$ & Catalytic efficiency $\left[\mathrm{M}^{-1} \mathrm{~min}^{-1}\right]$ \\
\hline OpuAA & $0.6 \pm 0.2$ & $1.1 \pm 0.1$ & 1833 \\
\hline OpuAA S45C & $0.7 \pm 0.1$ & $1.3 \pm 0.1$ & 1857 \\
\hline OpuAA G161C & $2.8 \pm 0.4$ & $0.8 \pm 0.1$ & 285 \\
\hline OpuAA S171C & Not determined & $0.08 \pm 0.01$ & Not calculated \\
\hline OpuAA F19W & $1.7 \pm 0.1$ & $1.5 \pm 0.1$ & 882 \\
\hline
\end{tabular}

Licenced copy. Copying is not permitted, except with prior permission and as allowed by law. (C) 2008 The Authors Journal compilation (C) 2008 Biochemical Society 
Table 2

\begin{tabular}{|l|l|l|l|}
\hline Functional state & S45C - S45C & S45C - G161C & G161C - G161C \\
\hline Static FRET & \multicolumn{3}{|l|}{} \\
\hline Nucleotide-free & $34 \%$ & $47 \%$ & $49 \%$ \\
& $57 \AA$ & $52 \AA$ & $51 \AA$ \\
\hline ATP & $39 \%$ & $79 \%$ & $65 \%$ \\
& $55 \AA$ & $41 \AA$ & $46 \AA$ \\
\hline ADP/Mg ${ }^{2+}$ & No transfer & No transfer & No transfer \\
\hline Time-resolved FRET & \multicolumn{3}{|l|}{} \\
\hline Nucleotide-free & $22 \pm 0.3 \mathrm{~min}$ & $20.7 \pm 0.1 \mathrm{~min}$ & $12.9 \pm 0.1 \mathrm{~min}$ \\
& $33 \%$ & $47 \%$ & $49 \%$ \\
& $59 \AA$ & $52 \AA$ & $51 \AA$ \\
\hline
\end{tabular}

Licenced copy. Copying is not permitted, except with prior permission and as allowed by law. (C) 2008 The Authors Journal compilation (C) 2008 Biochemical Society 


\section{Figure Legends:}

Figure 1:

(A): Homology model of the core domain OpuAA in the nucleotide-free state lacking the accessory domain. The model was generated using MODELLER 9v1 [31] as described in Materials and Methods. The helical domain is shown in light tan and the catalytic domain in yellow. The OpuAA-specific insertion between $\beta$-strand 1 and two is highlighted in grey. Conserved motifs are color-coded as follows: Walker A motif (blue), C-loop or ABCsignature motif (red), Walker B motif (magenta), D-loop (black) and H-loop (green). Positions of the single cysteine mutants are highlighted as spheres and labeled. (B): Sequence alignment of the accessory domains of OpuAA from B. subtilis (residues 277-418) and $L$. lactis (residues 271-408) containing a tandem CBS domain (residues 283- 335 and 342- 394 in the case of OpuAA from $B$. subtilis) with selected proteins containing CBS domains, for which a crystal structure is available. The two CBS domains in OpuAA from B. subtilis and L. lactis and IMPDH [41] are underlined. TM0892: open reading frame 0892 from Thermotoga maritium with unknown function (pdb entry 1VR9), TM0935: open reading frame 0935 from Thermotoga maritium with unknown function (pdb entry 1O50), TA0289: open reading frame 0289 from Thermoplasma acidophilum with unknown function (pdb entry 1PVM). (C): Homology model of the accessory domain of OpuAA from B. subtilis shown in cartoon representation. The tandem CBS domains are colored cyan (CBS1) and blue (CBS2), respectively. The $\mathrm{N}$ - and C-terminus, residues 277 and 418, respectively, of OpuAA are indicated. The model was derived from the crystal structure of TA0289 (pdb entry 1PVM) due to the high sequence identity (25\% identity and $51 \%$ homology) and obtained as described in Materials and Methods.

\section{Figure 2:}

Quantitative separation of labeled and non-labeled single cysteine mutants of OpuAA by anion exchange chromatography. OD at $216 \mathrm{~nm}$ (protein absorption) is shown in black and $\mathrm{OD}$ at $491 \mathrm{~nm}$ (OG absorption) and $591 \mathrm{~nm}$ (TR absorption) is shown as dashed lines, respectively. The applied $\mathrm{NaCl}$ concentration gradient is shown as a dashed, black line in the indicated concentration range. (A): OpuAA S45C-OG, (B): OpuAA S45C-TR, (C): OpuAA G161C-OG and (D): OpuAA G161C-TR. For further details see Materials and Methods.

\section{Figure 3:}

High-resolution MS analysis of OpuAA (A), OpuAA-OG S45C (B) and OpuAA-TR S45C (C). The arrow in (B) and (C) indicates the position of unlabelled OpuAA, which is below $2 \%$ according to an analysis of the peak area.

\section{Figure 4:}

Static FRET measurements of OpuAA S45C in the nucleotide-free (A), ATP-bound (B) and ADP-bound state (C) in the absence of $\mathrm{Mg}^{2+}$. In all three panels, the dashed, black line represents the sum of two individual FRET experiments, OpuAA S45C-OG/OpuAA wild type (molar ratio of 1:9) and wild type OpuAA/OpuAA S45C-TR (molar ration 1:9). The solid line shows the actual ERET experiments of OpuAA S45C-OG/OpuAA S45C-TR (molar ratio of 1:9). Acceptor concentration was always kept at $50 \mathrm{nM}$ and the corresponding dimers were prepared as described in Materials and Methods. The inset shows the difference spectra of the corresponding FRET experiments (OG and TR pairs). Buffer used: $10 \mathrm{mM}$ sodium phosphate $\mathrm{pH} 7.5,0.1 \mathrm{mM}$ EDTA, $1 \mathrm{M} \mathrm{NaCl}$.

\section{Figure 5:}

(A) Time-resolved FRET experiments. Dimers of the nucleotide-free state were prepared as described in Materials and Methods and chased by the addition of $10 \mathrm{mM}$ ADP/ $\mathrm{Mg}^{2+}$. 
Fluorescence was measured at $519 \mathrm{~nm}$ in $5 \mathrm{sec}$ intervals. From bottom to top: dimeric OpuAA S45C-OG/OpuAA S45C-TR (dashed trace), dimeric OpuAA G161C-OG/OpuAA S45C-TR (dotted trace), dimeric OpuAA G161C-OG/OpuAA G161C-TR (dotted-dashed trace) and monomeric OpuAA G161C-OG/OpuAA G161C-TR (black trace). Note that monomeric samples were measured at a roughly 4 fold higher concentration than the dimeric samples. Traces were analyzed according to equation (3). Buffer used: $10 \mathrm{mM}$ sodium phosphate $\mathrm{pH}$ 7.5, $0.1 \mathrm{mM}$ EDTA, $1 \mathrm{M} \mathrm{NaCl}$. (B) Time resolved transfer efficiencies were calculated according to equation (4) and extrapolated to $t=0$ to determine the initial transfer efficiencies, which are summarized in Table 3.

\section{Figure 6:}

Time-resolved, intrinsic tryptophan fluorescence of the OpuAA F19W mutant. The nucleotide-free, dimeric form of OpuAA was prepared as described for the wild type ATPase and ADP $(1 \mathrm{mM})$ was added at time point 0 in the absence of $\mathrm{Mg}^{2+}$. Tryptophan fluorescence was monitored at $350 \mathrm{~nm}$ over the indicated time frame and evaluated as described in Materials and Methods. Buffer used: $10 \mathrm{mM}$ sodium phosphate $\mathrm{pH}$ 7.5, 0.1 mM EDTA, $1 \mathrm{M}$ $\mathrm{NaCl}$.

Figure 7:

(A) Stereo-view of the FRET-derived homology model of nucleotide-free OpuAA. The accessory domains are shown in white while the canonical NBDs are given in yellow and light orange, respectively. Note that the accessory domain of MalK was used in the homology modeling, because proper location of the CBS domain, which is shown in Figure 1C, is not possible. FRET-derived distances between amino acids 45 (green) and 161 (dark grey) are highlighted by black dashes. The individual distances are given in Table 2. The Walker A motif is highlighted in blue and the ABC-signature motif in red. (B) Stereo-view of a structural superimposition of the FRET-derived homology model of OpuAA and the open conformation of the nucleotide-free form of MalK (pdb entry 1Q1E). Color-coding of OpuAA is identical to (A). Individual chains of MalK are shown in magenta and cyan, while the Walker $\mathrm{A}$ and $\mathrm{ABC}$-signature motifs are highlighted in blue and red, respectively. For structural superimpositions, the Walker A and B motifs were used. After improvement of the superimposition, the rmsd between monomer A of OpuAA and MalK was $1 \AA$ over $381 \mathrm{C} \alpha$ atoms.

\section{Figure 8:}

(A) Stereoview of the FRET-derived homology model of OpuAA in the ATP-bound state. ATP, which should be located between the Walker A (blue) and ABC signature (red) motifs of opposing monomers is not included in the model, because no structural knowledge of the exact position exists. The accessory domains, which adopt the structure of the MalK model, are depicted in white. The canonical NBDs are shown in cyan and magenta respectively. The anchors points (S45 and G161), which were used in the FRET studies, are highlighted in green and black, respectively and their distances indicated by dashed lines (exact values are given in Table 2). (B) Stereoview of the structural superimposition of the FRET-derived homology models of OpuAA in the ATP-bound state (cyan and magenta) and the nucleotidefree state (yellow and light orange). Color-coding of the conserved motifs is identical to (A). 


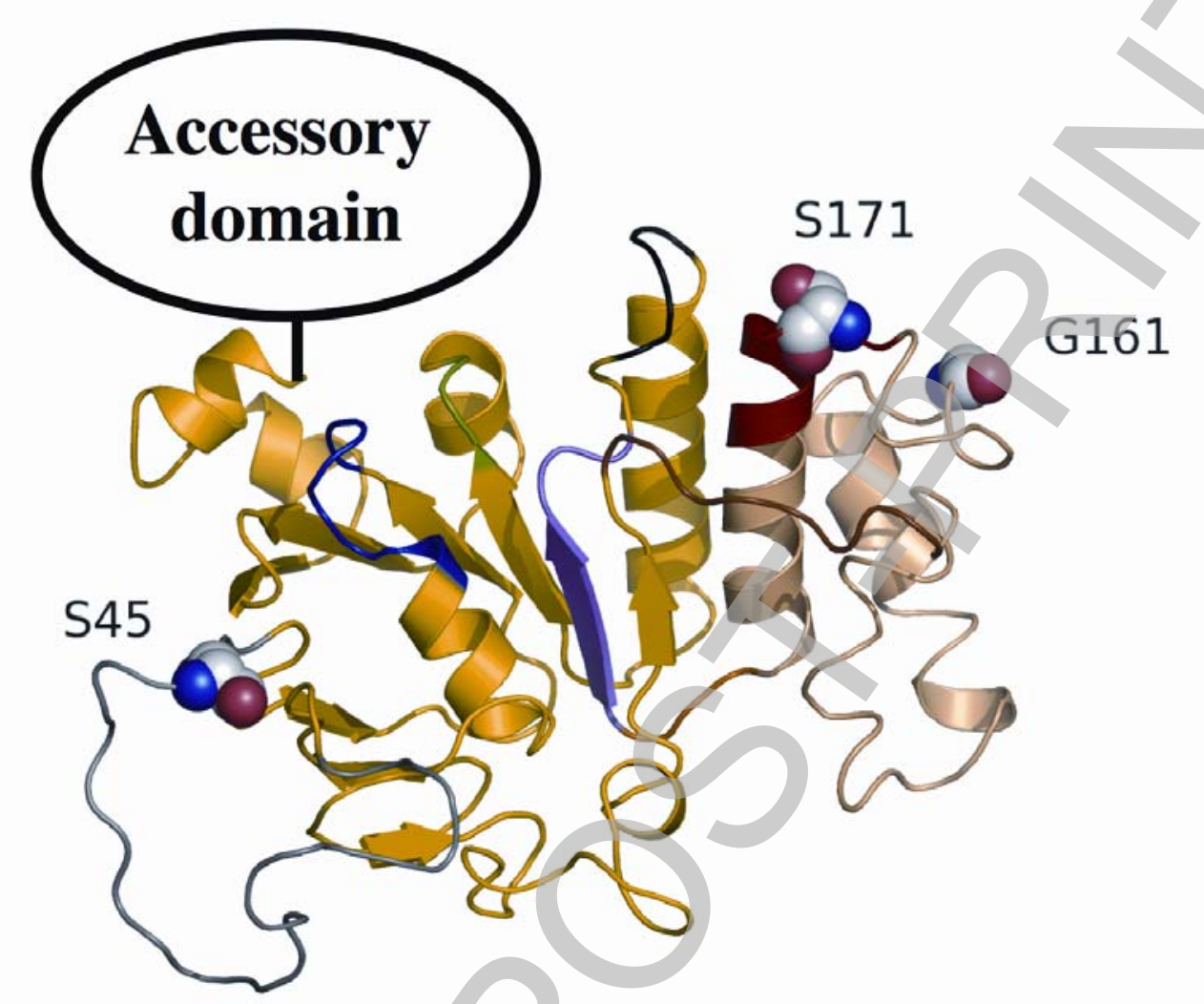

\section{Catalytic domain}

\section{Helical domain}

\section{B}

OpuAA B. subtilis OpuAA L. lactis IMPDH

TM0892

TM0935

TA0289

OpuAA B. subtilis OpuAA L. lactis IMPDH

TM0892

TM0935

TA0289

OpuAA B. subtilis OpuAA L. lactis IMPDH

TM0892

TM0935

TA0289
--- - - - - - EDVDLS---KVLTAGHIMKRAETVRIDK-GPRVALTLMKNLGISS 311 EILTNPANDYVKTFVEDVDLA---KVITAENIMIPALTTNIDVDGPSVALKKMQTEEVSS 308 -- ------EQAEEVRKVKRSENGVI IDPFFLTPEHK------VSEAEELMQRYRISG 123 ----MGSDKIHHHHHHMKVK---KWVTQDF PMVEESAT------VRECLHRMRQYQTNE 34 -----MGSDKIHHHHHHMKVKDVCKLISLKPTVVEEDTP------IEEIVDRILEDPVTR 37 --------VPRGGHMFMRVE---KIMNSNFKTVNWNTT------VFDAVKIMNENHLYG 42

IYAVD--KQKKLLGVIYASDAKKAAE------SDLSLQDILNTEFTTVPENTYLTEIFDV 363 LMAVD--RKRQFRGVVTSEQAIAARK------NNQSLKDVMTTDVGTVTKEMLVRDILPI 360 VPIVETLANRKLVGI ITNRDMRF ISD-----YNAPISEHMTSEHLVTAAVGTDLETAERI 178 CIVKD--REGHFRGVVNKEDLLDLDL-----DSSVFNKVSLPDFFVHEEDNITHALLLF 86 TVYVA--RDNKLVGMIPVMHLLKVS-------GFHFFGFIPKEELIRSSMKRLIAKNASE 88 LVVKD--DNGNDVGLLSERSIIKRF IPRNKKPDEVP IRLVMRKPIPKVKSDYDVKDVAAY 100

VSDANIPIAVVD-EKORMKGIVVRG----ALIGALAGNNEYINAEGTNEQTQDPSAQEVK 418 IYDAPTPLAVVD-DQGYLKGILIRG----IVLEALADIPDEVEE IEKEEEKND------- 408 LHEHRIEKLPLVDNSGRLSGLITIKDIEKVIEFPHAAKDEFGRLLVAAAVGVTSDTFERA 238 LEHOEPYLPVVD-EEMRLKGAVSLHDFLEALIEALAMDVPGIRFSVLLEDKPGELRKVVD 145 IMLDPVYVHMDTPLEEALKLMIDNNIQEMPVVDEKGEIVGDLNSLEILLALWKGREK--- 145 LSENGLERCAVVDDPGRVVGIVTLTDLSRYLSRASITDILLSHRTKDYQHLCPKCGVGVL 160 


\section{B] \\ Biochemical Journal Immediate Publication. Published on 06 Mar 2008 as manuscript BJ20071443}
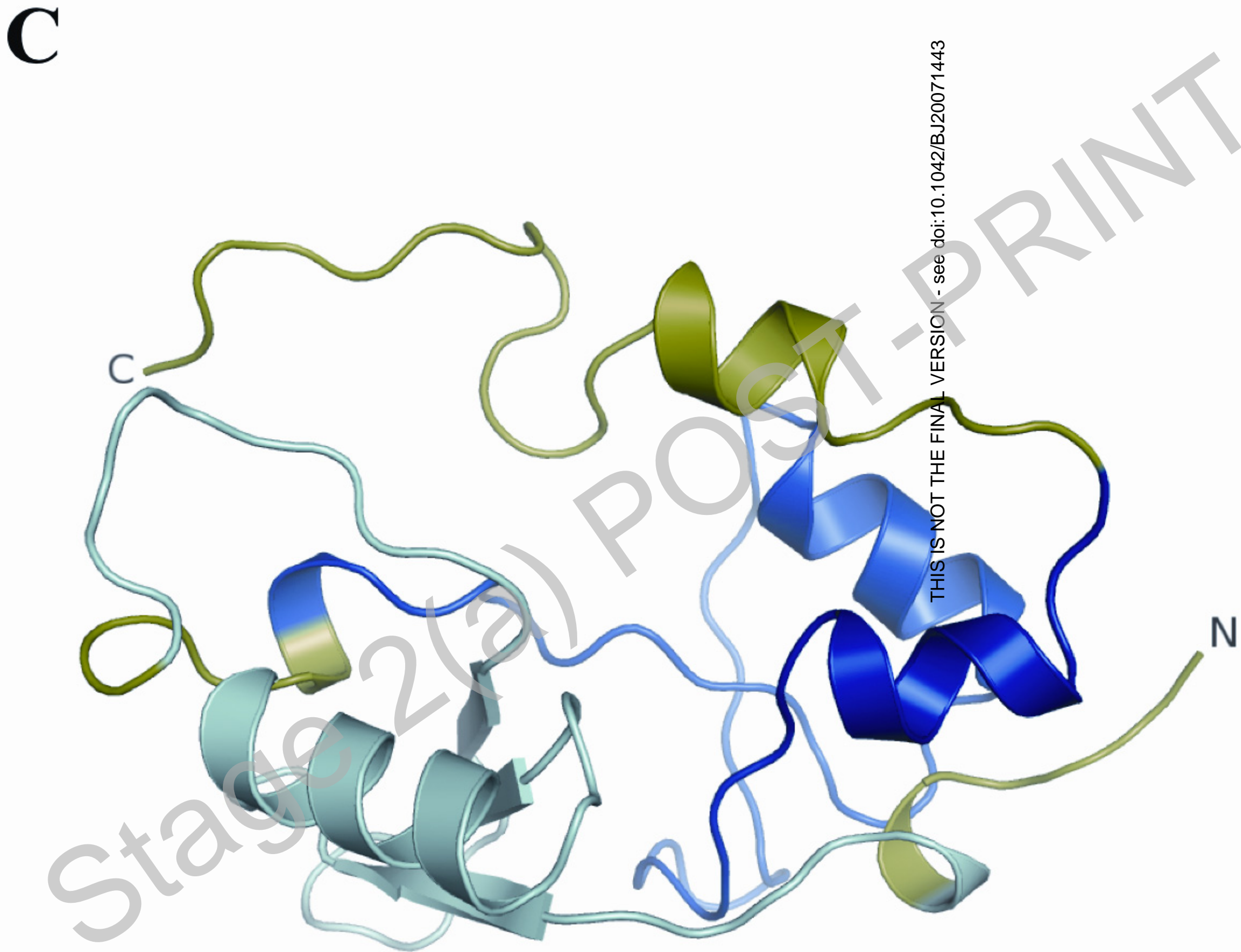

Licenced copy. Copying is not permitted, except with prior permission and as allowed by law. (C) 2008 The Authors Journal compilation (C) 2008 Biochemical Society 
A
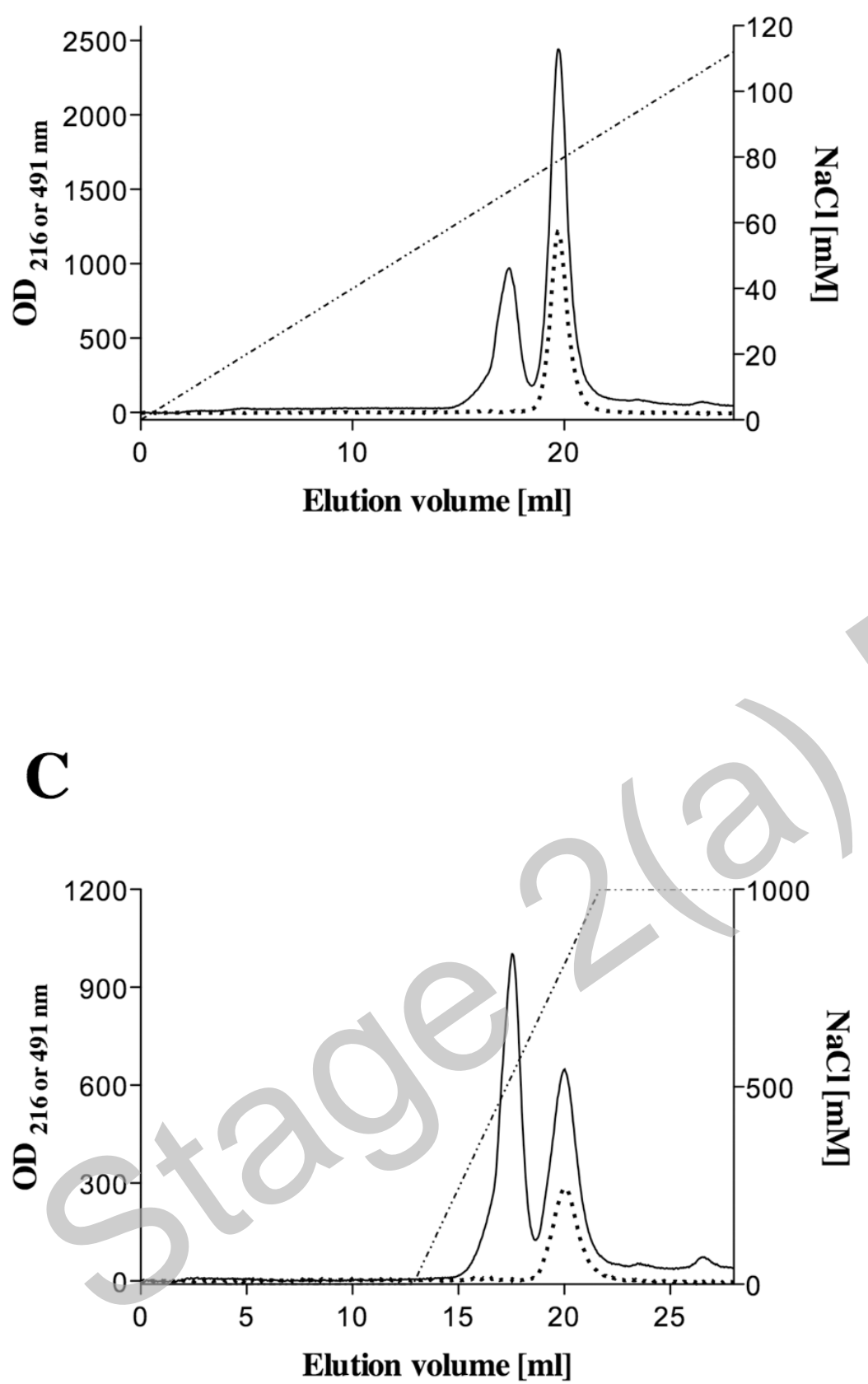

B
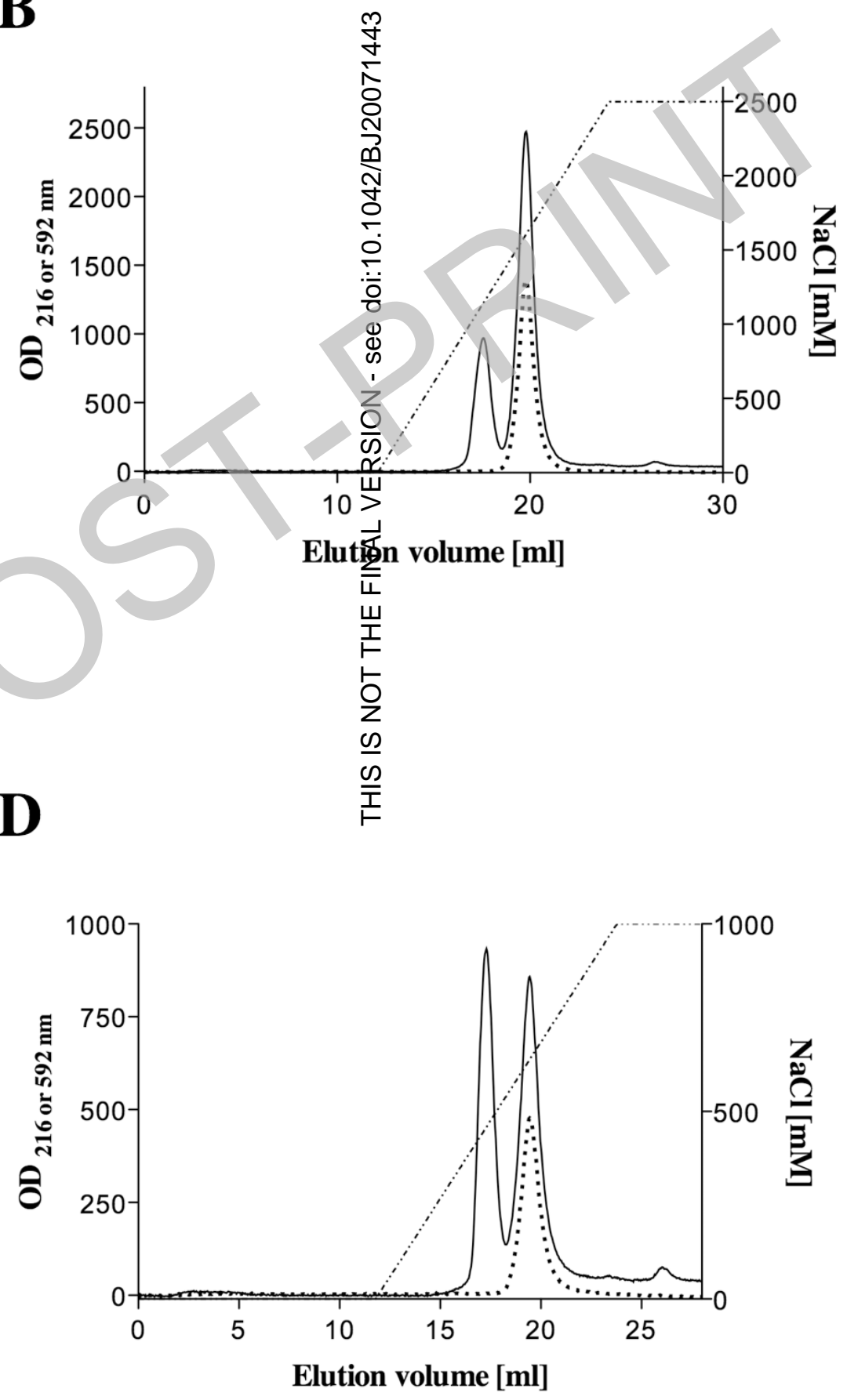

Licenced copy. Copying is not permitted, except with prior permission and as allowed by law. (c) 2008 The Authors Journal compilation (@ 2008 Biochemical Society 

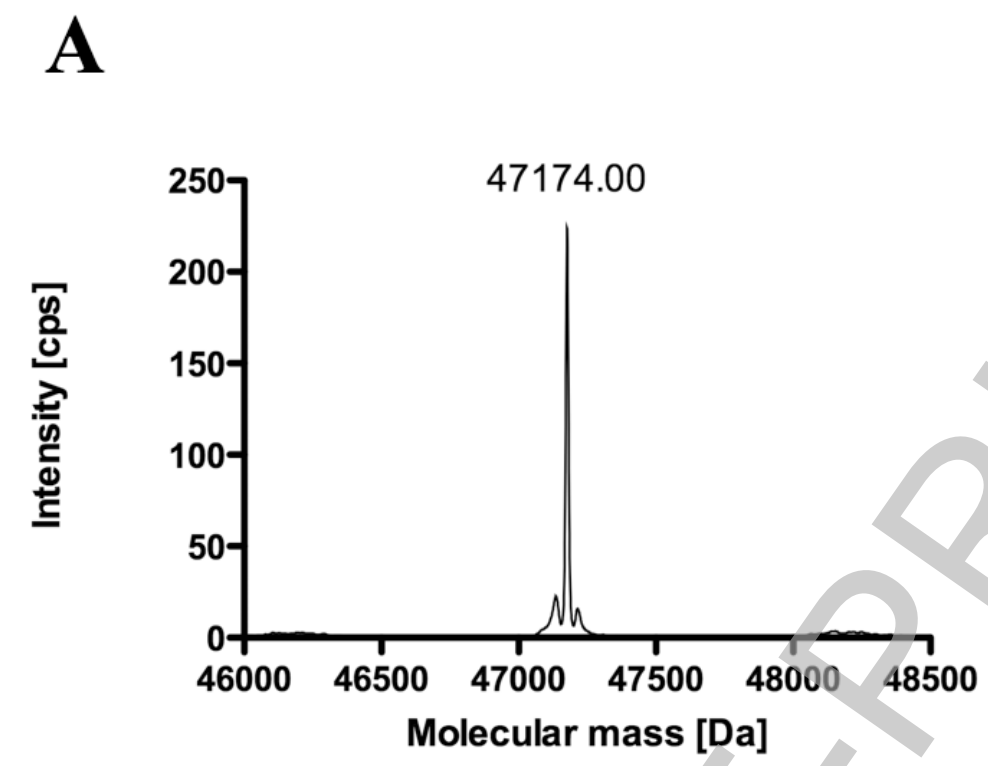

B

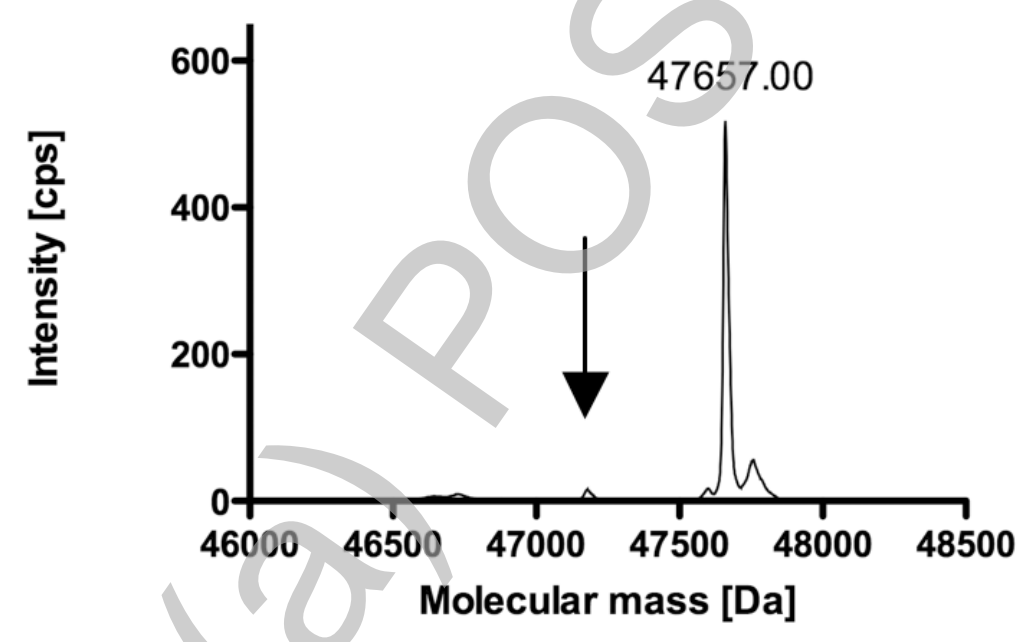

C

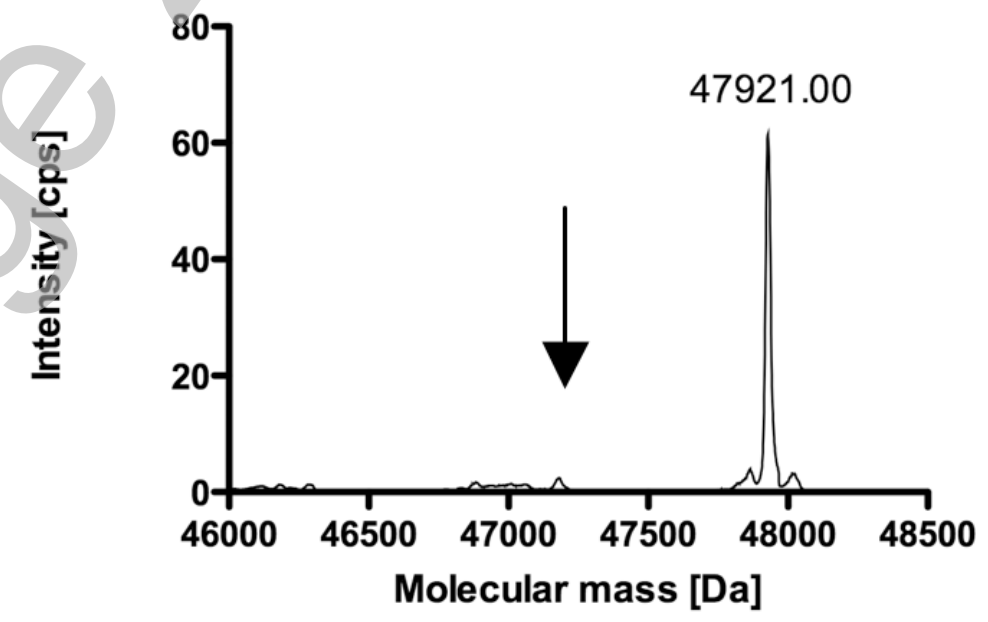

Licenced copy. Copying is not permitted, except with prior permission and as allowed by law. (C) 2008 The Authors Journal compilation ( 2008 Biochemical Society 

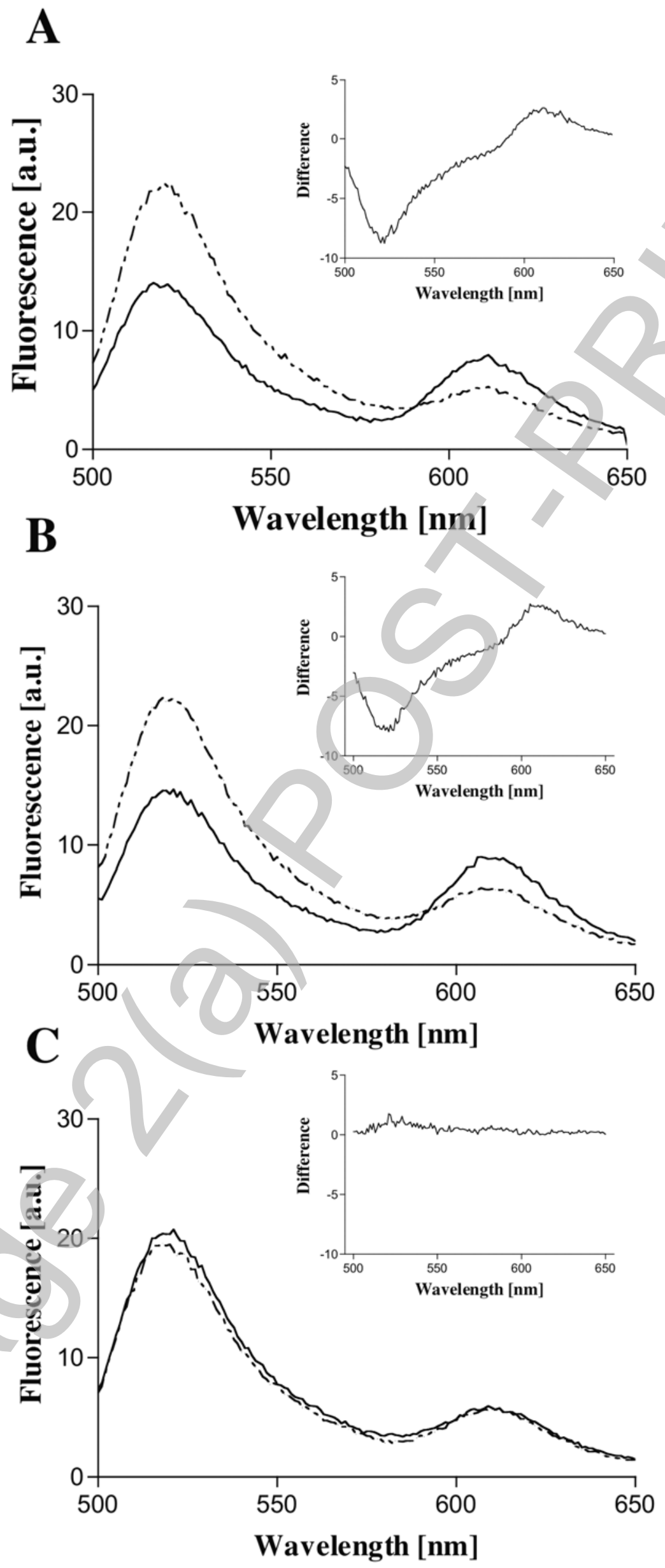

Licenced copy. Copying is not permitted, except with prior permission and as allowed by law. (C) 2008 The Authors Journal compilation (C) 2008 Biochemical Society 
A

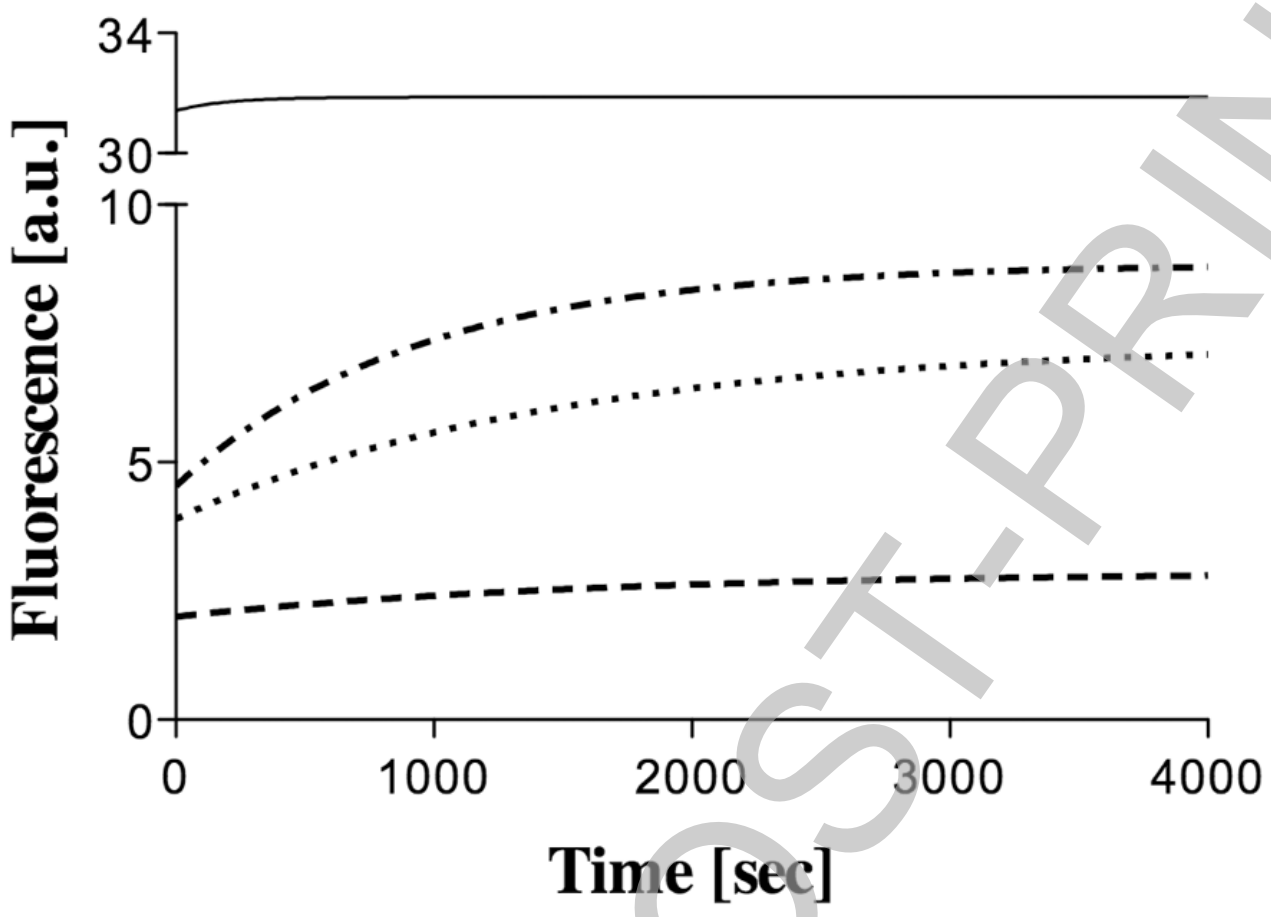

B

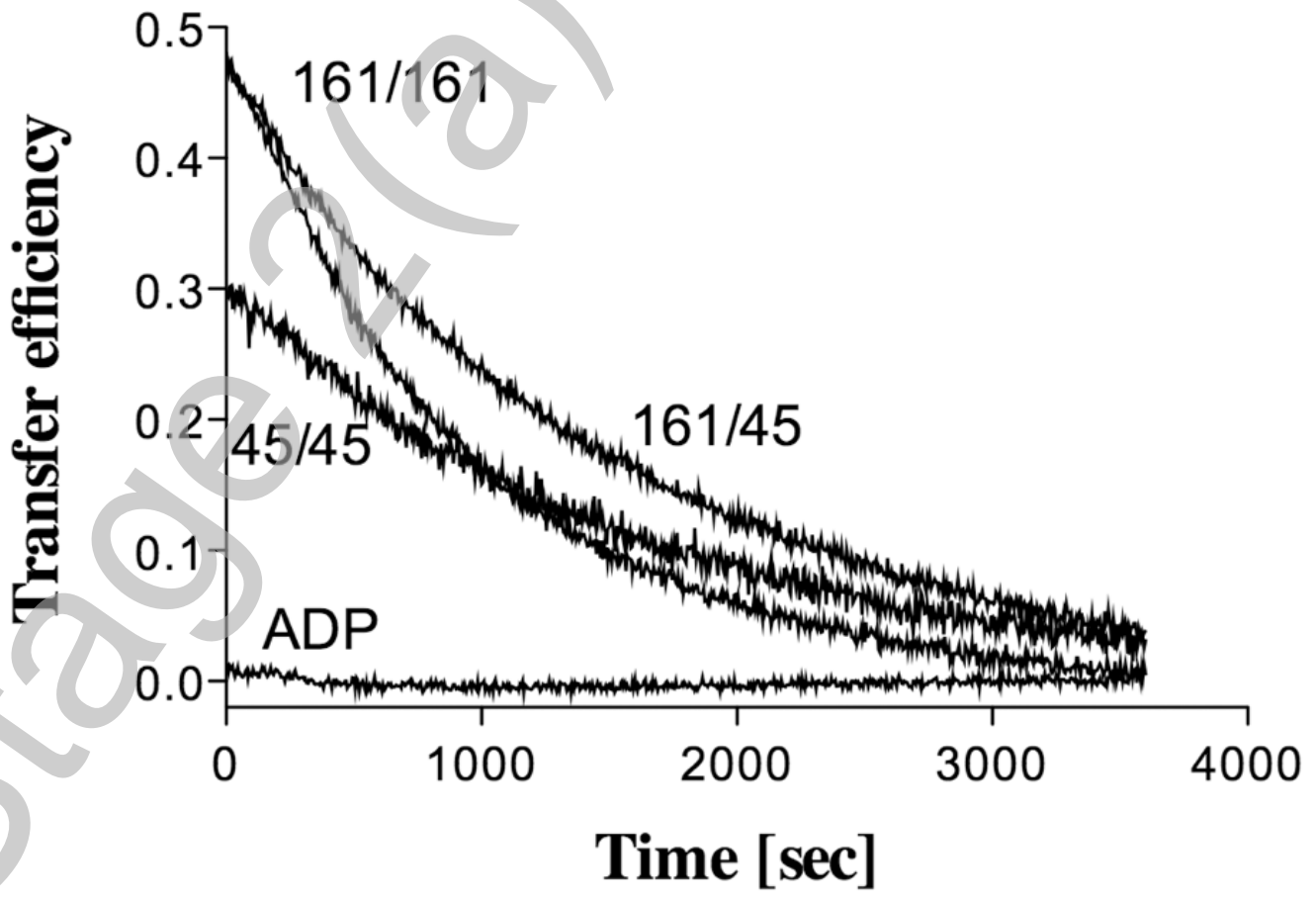

Licenced copy. Copying is not permitted, except with prior permission and as allowed by law. (C) 2008 The Authors Journal compilation (C) 2008 Biochemical Society 


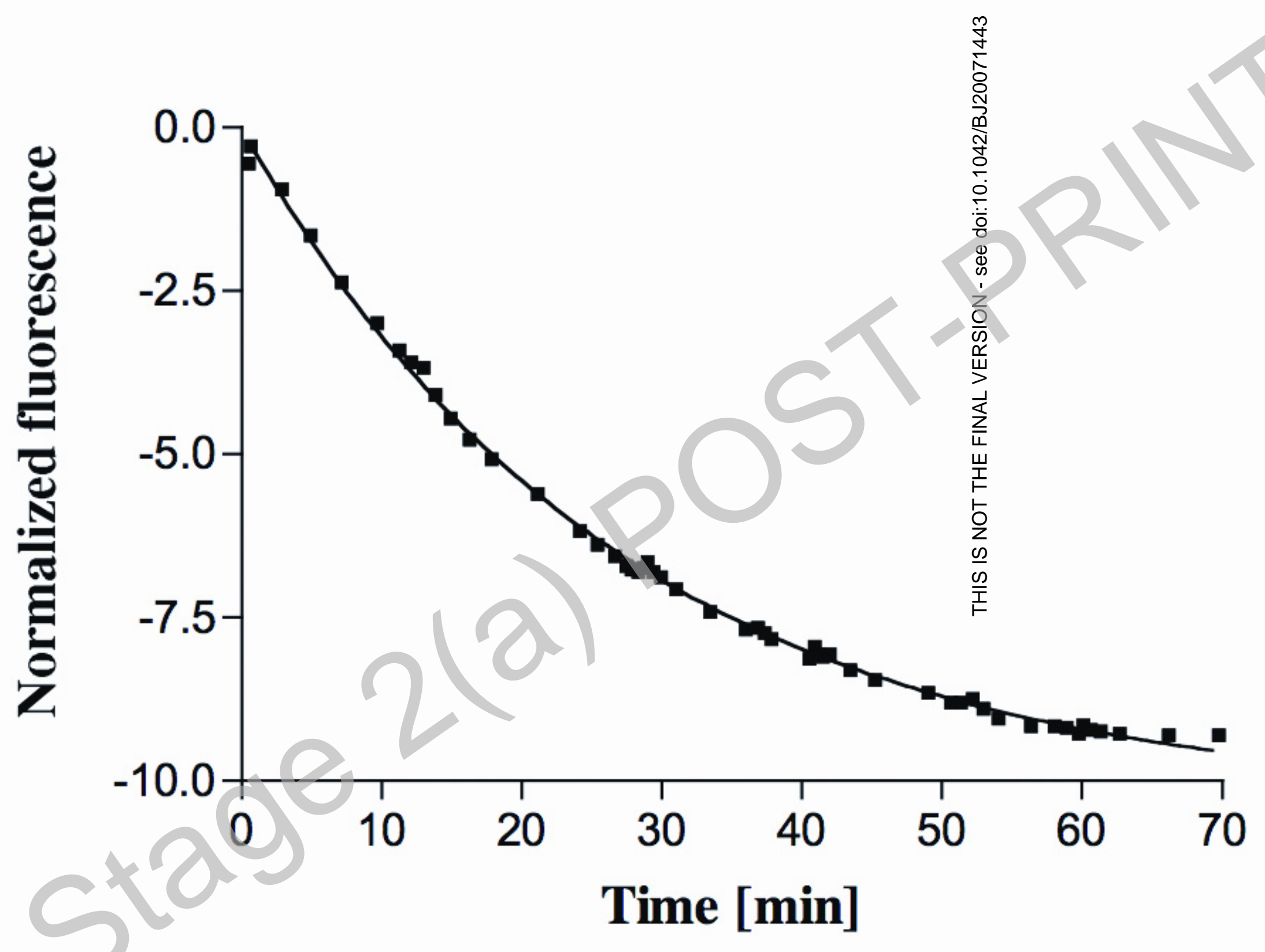



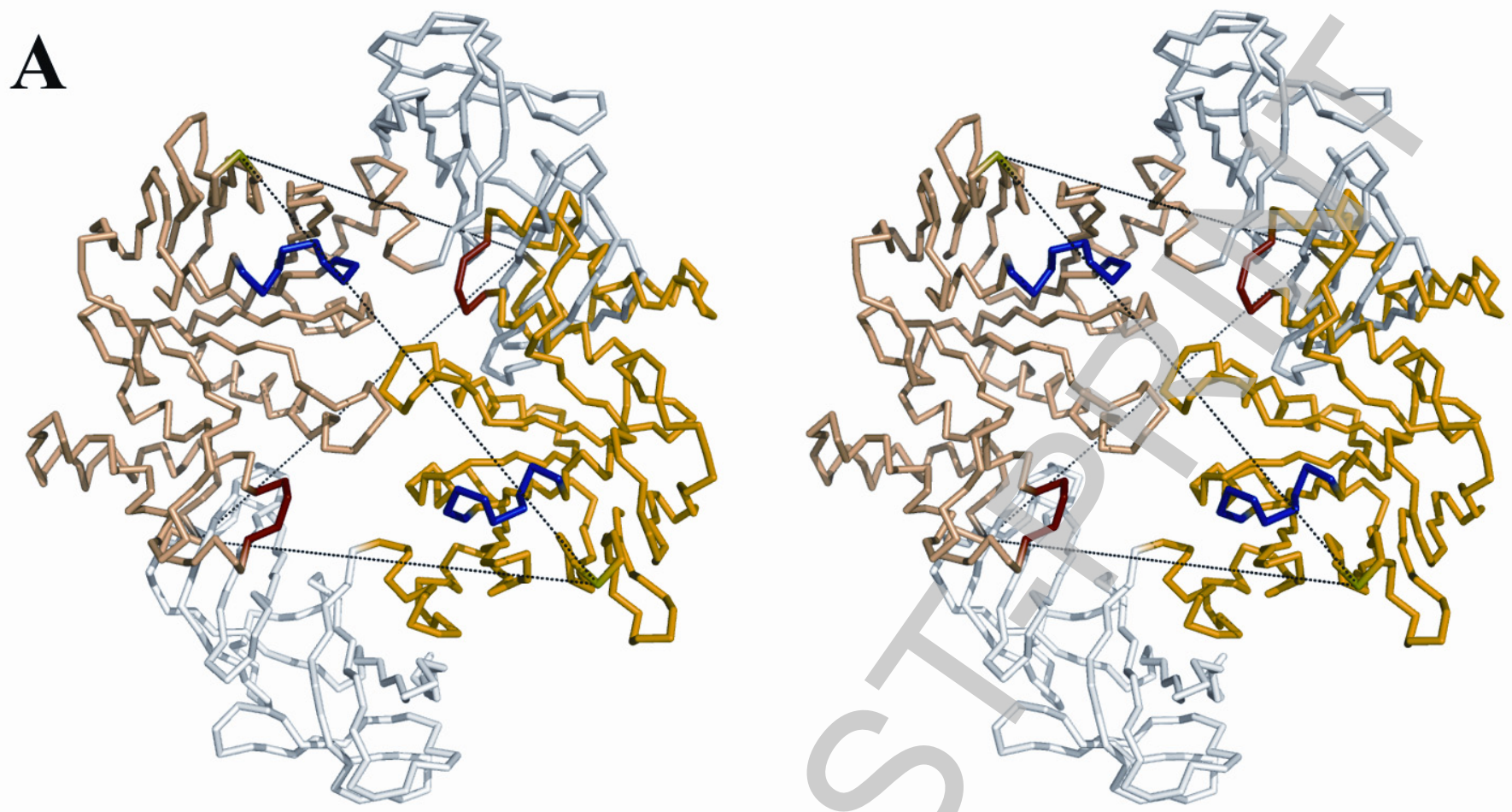

B
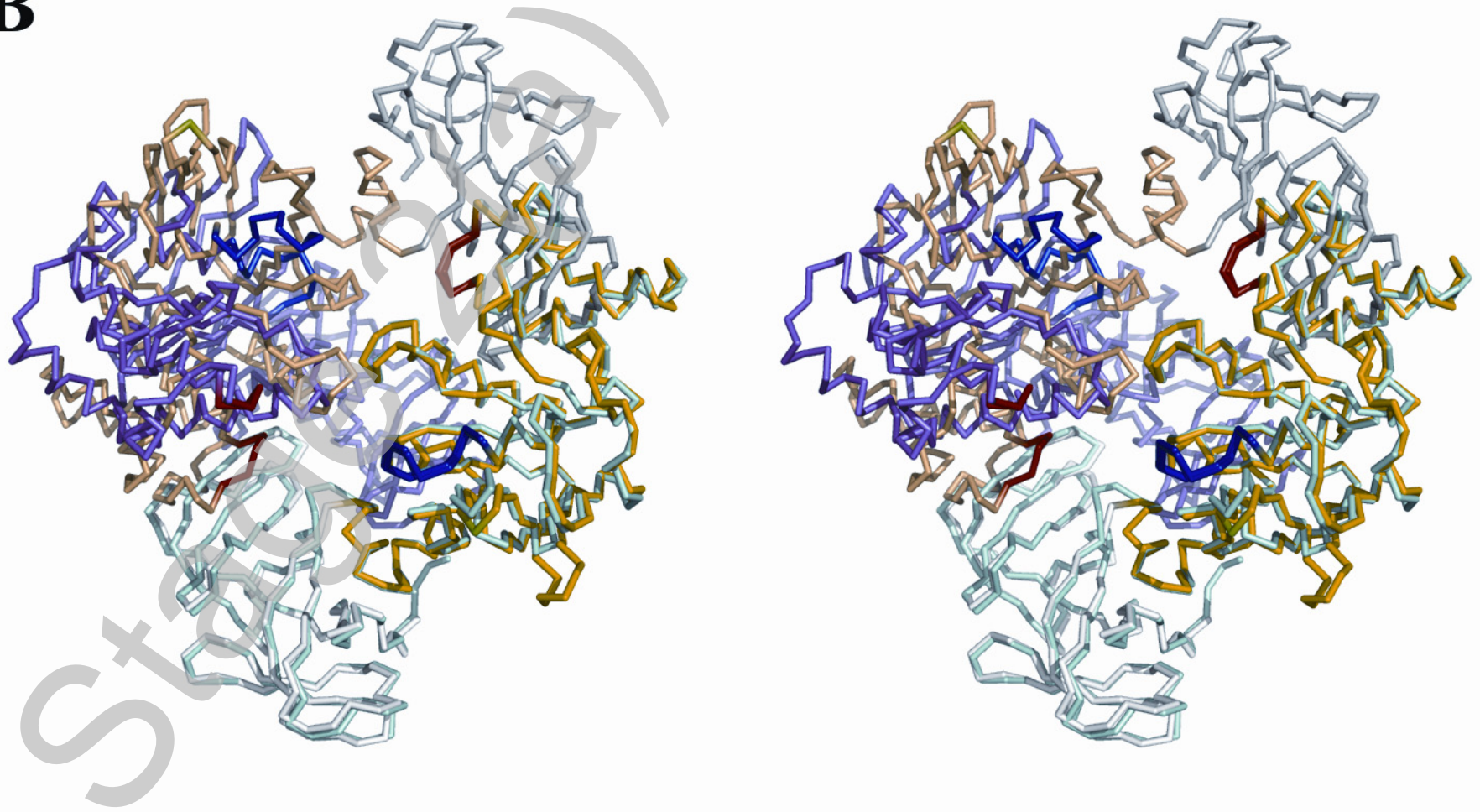

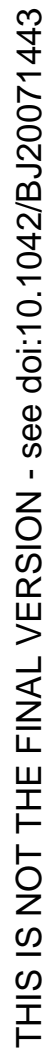

Licenced copy. Copying is not permitted, except with prior permission and as allowed by law. (c) 2008 The Authors Journal compilation (c) 2008 Biochemical Society 


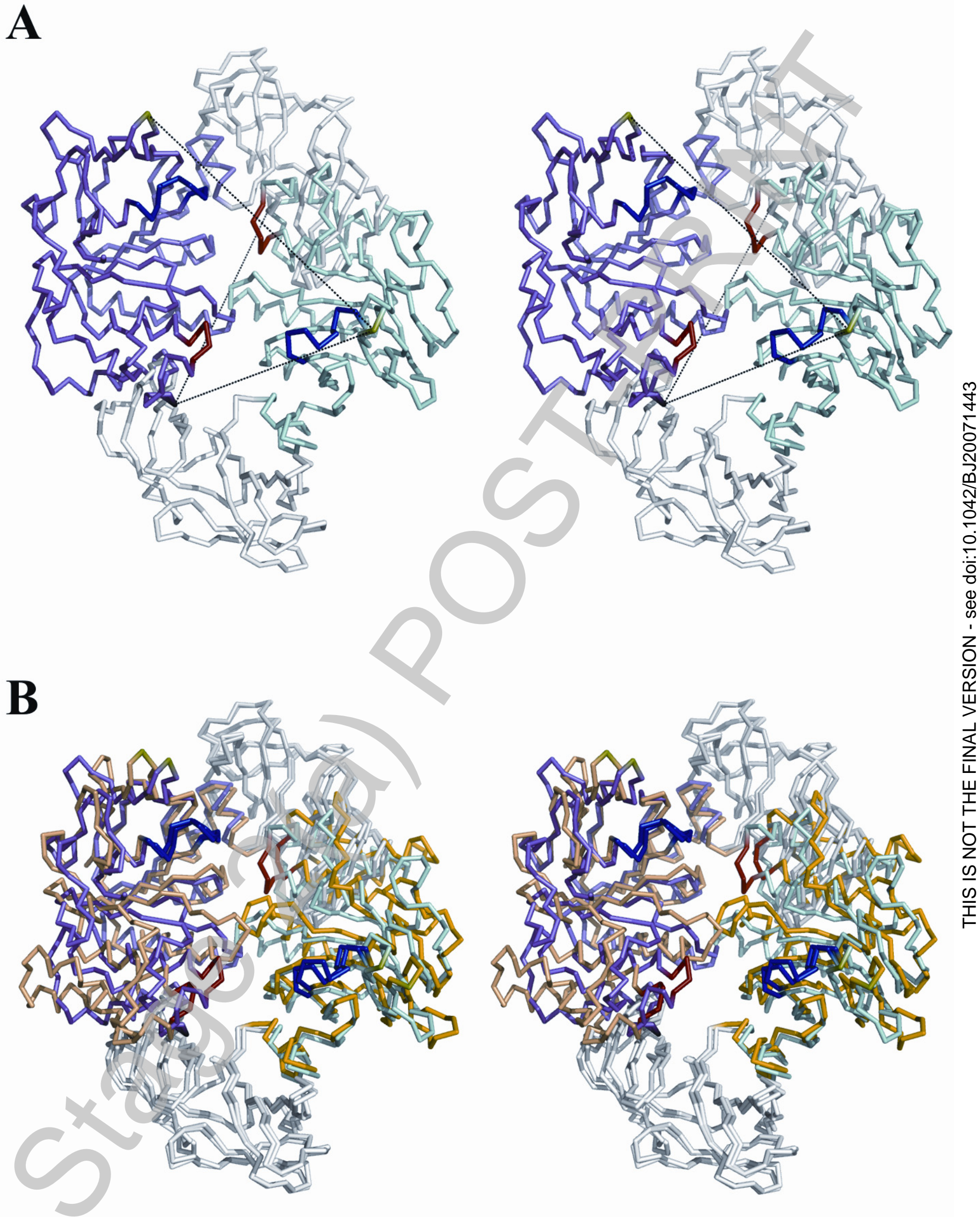

